\title{
Solutions and Drift Homogenization for a Class of Viscous
}

\section{Lake Equations in $L_{2}(\Omega)$}

Maoting Tong

Department of Mathematical Science, Xi'anJiaotong-Liverpool University, Suzhou, 215123, P.R.China, E-mail address:1760724097@qq.com

\begin{abstract}
In this paper we study solutions and drift homogenization for a class of viscous lake equations by using the method of semigroups of bounded operators. Suppose that the initial value $\left(t_{0}, u_{0}\right) \in U$, i.e., $u_{0}=u\left(t_{0}\right)$ for some Hölder continuous function $u$ on $[0, T]$ with smooth function value $u(t) \in D L_{2}(\Omega)_{\frac{1}{2}}$ satisfying $\partial_{j} u_{i}=0(i \neq j)$ and $b(x) \in C^{\infty}(\Omega)$. Then the initial value problem (2) for viscous lake equations has a unique smooth local strong solution. Using this result we study the drift homogenization for three-dimensional stationary Stokes equation in the usual sense $b D L_{2}(\Omega)$.
\end{abstract}

Mathematics Subject Classification (2010). Primary 35Q30, 35B27; Secondary 76N10,76M50,47D06

Keywords. viscous lake equations, Navier-Stokes equation, Existence and uniqueness, Semigroup of operators, Fractional powers.

* The author thanks the guidance and instruction of her tutor Andrew. Lin and Xinyao.Yang.

\section{(1) Introduction}

The viscous lake equations considered in this paper have the following equations (see the formula (1.3) in [17])

$$
\left\{\begin{array}{c}
\partial_{t}\left(b u^{\mu}\right)+\operatorname{div}\left(b u^{\mu} \otimes u^{\mu}\right)+A_{b}\left(u^{\mu}\right)+b \nabla p^{\mu}=0, x \in \Omega, t \in(0, T] \\
\operatorname{div}\left(b u^{\mu}\right)=0, x \in \Omega
\end{array}\right.
$$

for $(t, x) \in(0, T] \times \Omega$ with $\Omega \subset R^{3}$, a bounded domain with smooth boundary $\partial \Omega$ of class $C^{3}, \mu>0$ represents the eddy viscosity coefficient, $u^{\mu}=u^{\mu}\left(u_{1}^{\mu}(t, x), u_{2}^{\mu}(t, x), u_{3}^{\mu}(t, x)\right)$ stands for the three-dimensional fluid velocity, $p=p(t, x)$ is the pressure. Moreover, the depth $b(x)$ is a given function. We assume that the boundary is the only place where the depth can vanish, 
namely: there is a positive constant $M$ such that $0<b(x) \leq M$ in $\Omega . A_{b}$ is a viscous second order operator depending on $b$ which can satisfy the two expressions

$$
(i) A_{b}(\bullet)=-2 \mu \operatorname{div}(b D(\bullet)+2 b \operatorname{div}(\bullet) I), \quad(i i) A_{b}(\bullet)=-\mu b \Delta(\bullet)
$$

where $I$ is the $3 \times 3$ identity matrix and $D(\bullet)=\left(\nabla \bullet+(\nabla \bullet)^{t}\right) / 2$ is the deformation tensor. The equation (1) shows that the system does not describe incompressible flow, it is a constraint that plays a role similar to that played by the incompressibility condition for the incompressible Navier-Stokes system. We do not have an existence result concerning the solution of viscous shallow water equations with viscosity term given by $(i)$ until now.

We consider in this paper the well posedness of system (1)-(ii) with initial and boundary conditions which is given as follows

$$
\left\{\begin{array}{c}
\partial_{t} u^{\mu}+\left(u^{\mu} \bullet \nabla\right) u^{\mu}-\mu \Delta u^{\mu}+\nabla p^{\mu}=0, x \in \Omega, t \in\left(t_{0}, T\right] \\
\operatorname{div}\left(b u^{\mu}\right)=0, x \in \Omega \\
\left.u^{\mu}\right|_{t=t_{0}}=u_{0}^{\mu}, x \in \Omega \\
u^{\mu}(t, x)=0, x \in \partial \Omega
\end{array}\right.
$$

The existence, uniqueness and regularity properties of solutions for the viscous lake equations are extensively studied. There is an extensive literature on the solvability of the initial value problem for viscous lake equations. The terms and symbols in this paper are the same as [18]. For some narratives and background, please refer to [18].

In case when $b(x)$ is a constant, system (2) becomes similar to the classical 3D incompressible Navier-Stokes equations with the external force $f=0$ as follows

$$
\left\{\begin{array}{c}
\frac{\partial u}{\partial t}=\Delta u-\nabla p-(u \bullet \nabla) u, x \in \Omega, t \in\left(t_{0}, T\right] \\
\nabla \bullet u \equiv \operatorname{div} u=0 \\
\left.u\right|_{\partial \Omega}=0, t \in\left(t_{0}, T\right] \\
\left.u\right|_{t=t_{0}}=u_{0}, x \in \Omega
\end{array}\right.
$$

Let $L_{2}(\Omega)$ be the Hilbert space of real vector functions in $L^{2}(\Omega)$. That is

$$
L_{2}(\Omega)=\left\{u: \Omega \rightarrow R^{3}, u=\left(u_{1}, u_{2}, u_{3}\right), u_{i} \in L^{2}(\Omega)(i=1,2,3)\right\} .
$$

For $u=\left(u_{1}, u_{2}, u_{3}\right), v=\left(v_{1}, v_{2}, v_{3}\right) \in L_{2}(\Omega)$, we define the norm and the inner

$$
\|u\|_{L_{2}(\Omega)}=\left(\sum_{i=1}^{3}\left\|u_{i}\right\|_{L^{2}(\Omega)}^{2}\right)^{\frac{1}{2}},(u, v)=\sum_{i=1}^{3}\left(u_{i}, v_{i}\right)
$$

then $L_{2}(\Omega)$ is also a Hilbert space. The set of all real vector functions $u$ such that div $b u=0$ 
and $u \in C_{0}^{\infty}(\Omega)$ is denoted by $C_{0, b}^{\infty}(\Omega)$. Let $b D L_{2}(\Omega)$ be the closure of $C_{0, b}^{\infty}(\Omega)$ in $L_{2}(\Omega)$. The set of all real vector functions $u$ such that $\operatorname{div} u=0$ and $u \in C_{0}^{\infty}(\Omega)$ is denoted by $C_{0, \sigma}^{\infty}(\Omega)$. Let $D L_{2}(\Omega)$ be the closure of $C_{0, \sigma}^{\infty}(\Omega)$ in $L_{2}(\Omega)$. In the case when $b$ is a constant $b D L_{2}(\Omega)=D L_{2}(\Omega)$. Similarly to p.270 in [4] we can prove that if $u \in C^{\infty}(\Omega)$ then $u \in b D L_{2}(\Omega)$ if and only if $d i v b u=0$ in $\Omega$ and $u_{n}=0$ on $\partial \Omega$.

(see lemma 1) We will see in lemma 7 that if $b \in C^{\infty}(\Omega)$ then

$$
C_{0, \sigma}^{\infty}(\Omega) \subseteq C_{0, b}^{\infty}(\Omega) \subseteq L_{2}(\Omega), \quad D L_{2}(\Omega) \subseteq b D L_{2}(\Omega) \subseteq L_{2}(\Omega)=W^{0,2}(\Omega),
$$

and

$$
\begin{gathered}
\|\bullet\|_{D L_{2}(\Omega)}=\|\bullet\|_{b D L_{2}(\Omega)}=\|\bullet\|_{L_{2}(\Omega)}, \\
L_{2}(\Omega)=D L_{2}(\Omega) \oplus\left(D L_{2}(\Omega)\right)^{\perp}=b D L_{2}(\Omega) \oplus\left(b D L_{2}(\Omega)\right)^{\perp} .
\end{gathered}
$$

From [4] and [9] we have $\left(D L_{2}(\Omega)\right)^{\perp}=\left\{\nabla h ; h \in W^{1,2}(\Omega)\right\}$ and $\left(b D L_{2}(\Omega)\right)^{\perp} \supseteq$ $\left\{b \nabla h ; h \in W^{1,2}(\Omega)\right\}$. (see lemma 2) Let $P$ be the orthogonal projection from $L_{2}(\Omega)$ onto $b D L_{2}(\Omega) . A=P \Delta$ is called the Stokes operator. Since $\nabla p=b \nabla h$ has a solution $h \in W^{1,2}(\Omega)$ for $b \in C^{\infty}(\Omega)$. By applying $P$ to the first equation of (3) and taking account of the other equations, we are let the following abstract initial value problem, Pr. II

$$
\left\{\begin{array}{c}
\frac{d u}{d t}=P \Delta u+F u, t \in\left(t_{0}, T\right] \\
\left.u\right|_{t=t_{0}}=u_{0}, x \in \Omega
\end{array}\right.
$$

where $F u=-P(u \bullet \nabla) u$ and $P \nabla p=0$. We consider equation (5) in integral form Pr.III

$$
u(t)=e^{t P \Delta} u_{0}+\int_{t_{0}}^{t} e^{(t-s) P \Delta} F u(s) d s .
$$

For $u=\left(u_{1}, u_{2}, u_{3}\right) \in L_{2}(\Omega)$ we define $\Delta u=\left(\Delta u_{1}, \Delta u_{2}, \Delta u_{3}\right)$. We take a rectangular coordinate system. If $u(t, x)=\left(u_{1}(t, x), u_{2}(t, x), u_{3}(t, x)\right)$ is a velocity field, define $\nabla u=\left(\frac{\partial u_{1}}{\partial x_{1}}\right.$ $\left.\frac{\partial u_{2}}{\partial x_{2}}, \frac{\partial u_{3}}{\partial x_{3}}\right)$. Since the operator $-\nabla=-\sum_{i=1}^{3} \frac{\partial^{2}}{\partial x_{i}^{2}}$ is strongly elliptic of order 2. Theorem7.3.6 
in [11] implies that $\Delta$ is the infinitesimal generator of an analytic semigroup of contractions on $L^{2}(\Omega)$ with $D(-\Delta)=H^{2}(\Omega) \cap H_{0}^{1}(\Omega)$. Hence $\Delta$ is also the infinitesimal generator of an analytic semigroup of contraction on $L_{2}(\Omega)$ with $D(-\Delta)=H_{2}(\Omega) \cap H_{1,0}(\Omega)$, where $H_{2}(\Omega)$ and $H_{1,0}(\Omega)$ are the Sobolev spaces of vector value in $H^{2}(\Omega)$ and $H_{0}^{1}(\Omega)$ respectively. We will prove that $\Delta$ is also the infinitesimal generator of an analytic semigroup of contraction on $b D L_{2}(\Omega)$.

\section{(2) Some lemmas}

For $u \in L_{2}(\Omega)$, if $d i v \quad b u=0$ then $u$ is called $b$ divergence free.

Lemma 1. If $u \in C^{\infty}(\Omega)$ then $u \in b D L_{2}(\Omega)$ implies div $b u=0$.

Proof. Suppose that $u=\left(u_{1}, u_{2}, u_{3}\right) \in C^{\infty}(\Omega) \cap b D L_{2}(\Omega)$. Then there exists a sequence $\left\{u^{n} \in C_{0, b}^{\infty}(\Omega): n=1,2, \ldots\right\}$ such that $u=\lim _{n \rightarrow \infty} u^{n}$, that is $\lim _{n \rightarrow \infty}\left\|u-u^{n}\right\|_{b D L_{2}(\Omega)}=0$ uniformly on $\Omega$ and $\lim _{n \rightarrow \infty}\left\|u_{i}-u_{i}^{n}\right\|_{L^{2}(\Omega)}=0$ uniformly on $\Omega$ for $i=1,2,3$ where $\operatorname{div} b u^{n}=0$. It follows from Theorem 7.16 in [12] that

$$
\lim _{n \rightarrow \infty}\left[\int_{\Omega}\left|u_{i}(x)-u_{i}^{n}(x)\right|^{2} d x\right]^{\frac{1}{2}}=\left[\int_{\Omega} \lim _{n \rightarrow \infty}\left|u_{i}(x)-u_{i}^{n}(x)\right| d x\right]^{\frac{1}{2}}=0
$$

And so $\lim _{n \rightarrow \infty}\left|u_{i}(x)-u_{i}^{n}(x)\right|=0$ and $\lim _{n \rightarrow \infty} u_{i}^{n}(x)=u_{i}(x)$ uniformly on $\Omega$ for $i=1,2,3$. (see Theorem 1.39 in [14]) From the proof of Theorem 7.11 in [13] it follows that

$$
\begin{aligned}
& \text { divbu }=\sum_{i=1}^{3} \frac{\partial b u_{i}}{\partial x_{i}}=\sum_{i=1}^{3} \frac{\partial\left(b \lim _{n \rightarrow \infty} u_{i}^{n}\right)}{\partial x_{i}}=\sum_{i=1}^{3}\left(\frac{\partial b}{\partial x_{i}} \lim _{n \rightarrow \infty} u_{i}^{n}+b \frac{\partial}{\partial x_{i}} \lim _{n \rightarrow \infty} u_{i}^{n}\right) \\
& =\sum_{i=1}^{3}\left[\lim _{n \rightarrow \infty} \frac{\partial b}{\partial x_{i}} u_{i}^{n}+b \lim _{x_{i} \rightarrow x_{i 0}} \frac{\lim _{n \rightarrow \infty} u_{i}^{n}\left(x_{i}\right)-\lim _{n \rightarrow \infty} u_{i}^{n}\left(x_{i 0}\right)}{x_{i}-x_{i 0}}\right] \\
& =\sum_{i=1}^{3}\left[\lim _{n \rightarrow \infty} \frac{\partial b}{\partial x_{i}} u_{i}^{n}+b \lim _{n \rightarrow \infty} \lim _{x_{i} \rightarrow x_{i 0}} \frac{u_{i}^{n}\left(x_{i}\right)-u_{i}^{n}\left(x_{i 0}\right)}{x_{i}-x_{i 0}}\right] \\
& =\sum_{i=1}^{3}\left[\lim _{n \rightarrow \infty} \frac{\partial b}{\partial x_{i}} u_{i}^{n}+b \lim _{n \rightarrow \infty} \frac{\partial u_{i}^{n}}{\partial x_{i}}\right] \\
& =\lim _{n \rightarrow \infty} \sum_{i=1}^{3}\left[\frac{\partial b u_{i}^{n}}{\partial x_{i}}\right] \\
& =0
\end{aligned}
$$

This is to say $d i v b u=0$. 
Lemma 2. $\left(b D L_{2}(\Omega)\right)^{\perp} \supseteq\left\{b \nabla h ; h \in W^{1,2}(\Omega)\right\}$.

Proof. Suppose $u \in C_{0, b}^{\infty}(\Omega)$. Then from integration by parts we have for $h \in W^{1,2}(\Omega)$

$$
\begin{aligned}
& (u, b \nabla h)=\sum_{i=1}^{3}\left(u_{i}, \frac{b \partial h}{\partial x_{i}}\right)=\sum_{i=1}^{3} \int_{\Omega} b u_{i} \frac{\partial h}{\partial x_{i}} d x=\int_{\partial \Omega}\left(\sum_{i=1}^{3} b u_{i} h\right) d x-\int_{\Omega}\left(\sum_{i=1}^{3} \frac{\partial\left(b u_{i}\right)}{\partial x_{i}} h\right) d x \\
& =-\int_{\Omega}\left(\sum_{i=1}^{3} \frac{\partial\left(b u_{i}\right)}{\partial x_{i}}\right) h d x=-\int_{\Omega} \operatorname{div}(b u) h d x=0 .
\end{aligned}
$$

If $u \in b D L_{2}(\Omega)$, then there exists a sequence $\left\{u^{n} \in C_{0, b}^{\infty}(\Omega): n=1,2, \ldots\right\}$ such that $u=\lim _{n \rightarrow \infty} u^{n}$ uniformly on $\Omega$. Then

$$
(u, b \nabla h)=\left(\lim _{n \rightarrow \infty} u^{n}, b \nabla h\right)=\lim _{n \rightarrow \infty}\left(u^{n}, b \nabla h\right)=0 .
$$

Lemma 3. For every $u \in L_{2}(\Omega)$, div $u=0$ if and only if $\operatorname{div}(\lambda I-\Delta) u=0$ for

$$
\lambda \in \Sigma_{\vartheta}=\{\lambda: \vartheta-\pi<\arg \lambda<\pi-\vartheta,|\lambda| \geq r\}
$$

where $0<\vartheta<\pi / 2$.

Proof. Let $u=\left(u_{1}, u_{2}, u_{3}\right) \in L_{2}(\Omega)$. Then

$$
\begin{aligned}
\Delta u & =\left(\frac{\partial^{2} u_{1}}{\partial x_{1}^{2}}+\frac{\partial^{2} u_{1}}{\partial x_{2}^{2}}+\frac{\partial^{2} u_{1}}{\partial x_{3}^{2}}, \frac{\partial^{2} u_{2}}{\partial x_{1}^{2}}+\frac{\partial^{2} u_{2}}{\partial x_{2}^{2}}+\frac{\partial^{2} u_{2}}{\partial x_{3}^{2}}, \frac{\partial^{2} u_{3}}{\partial x_{1}^{2}}+\frac{\partial^{2} u_{3}}{\partial x_{2}^{2}}+\frac{\partial^{2} u_{3}}{\partial x_{3}^{2}}\right), \\
\operatorname{div}(\Delta u) & =\frac{\partial^{3} u_{1}}{\partial x_{1}^{3}}+\frac{\partial^{3} u_{1}}{\partial x_{1} \partial x_{2}^{2}}+\frac{\partial^{3} u_{1}}{\partial x_{1} \partial x_{3}^{2}}+\frac{\partial^{3} u_{2}}{\partial x_{2} \partial x_{1}^{2}}+\frac{\partial^{3} u_{2}}{\partial x_{2}^{3}}+\frac{\partial^{3} u_{2}}{\partial x_{2} \partial x_{3}^{2}}+\frac{\partial^{3} u_{3}}{\partial x_{3} \partial x_{1}^{2}}+\frac{\partial^{3} u_{3}}{\partial x_{3} \partial x_{2}^{2}}+\frac{\partial^{3} u_{3}}{\partial x_{3}^{3}} \\
& =\frac{\partial^{2}}{\partial x_{1}^{2}}\left(\frac{\partial u_{1}}{\partial x_{1}}+\frac{\partial u_{2}}{\partial x_{2}}+\frac{\partial u_{3}}{\partial x_{3}}\right)+\frac{\partial^{2}}{\partial x_{2}^{2}}\left(\frac{\partial u_{1}}{\partial x_{1}}+\frac{\partial u_{2}}{\partial x_{2}}+\frac{\partial u_{3}}{\partial x_{3}}\right)+\frac{\partial^{2}}{\partial x_{3}^{2}}\left(\frac{\partial u_{1}}{\partial x_{1}}+\frac{\partial u_{2}}{\partial x_{2}}+\frac{\partial u_{3}}{\partial x_{3}}\right) \\
= & \left(\frac{\partial^{2}}{\partial x_{1}^{2}}+\frac{\partial^{2}}{\partial x_{2}^{2}}+\frac{\partial^{2}}{\partial x_{3}^{2}}\right)\left(\frac{\partial u_{1}}{\partial x_{1}}+\frac{\partial u_{2}}{\partial x_{2}}+\frac{\partial u_{3}}{\partial x_{3}}\right)=\Delta(\text { divu }) .
\end{aligned}
$$

So we have

$$
\operatorname{div}[(\lambda I-\Delta) u]=(\lambda I-\Delta)(\operatorname{div} u) .
$$

From (7) it is clear that $\operatorname{div} u=0$ implies that $\operatorname{div}(\lambda I-\Delta) u=0$.

Since $-\Delta$ is a strongly elliptic operator of order 2 on $\Omega$. From Theorem 7.3.2 in [11] it follows that there exist constant $C>0, r \geq 0$ and $0<\vartheta<\pi / 2$ such that 


$$
\|u\|_{L_{2}(\Omega)} \leq \frac{C}{|\lambda|}\|(\lambda I-\Delta) u\|_{L_{2}(\Omega)}
$$

for $u \in D(\Delta)=H_{2}(\Omega) \cap H_{1,0}(\Omega) \subset L_{2}(\Omega)$ and

$$
\lambda \in \Sigma_{\vartheta}=\{\lambda: \vartheta-\pi<\arg \lambda<\pi-\vartheta,|\lambda| \geq r\}
$$

From (8) it follows that for every $\lambda \in \Sigma_{\vartheta}$ the operator $\lambda I-\Delta$ is injective from $D(\Delta)$ into $L_{2}(\Omega)$. From (7) it follows that $\operatorname{div}(\lambda I-\Delta) u=0$ implies that $\operatorname{div} u=0$.

Lemma 4. (1.5.12 in [6]) Let $\{T(t): t \geq 0\}$ be a $C_{0}$-semigroup on a Banach space $X$. If $Y$ is a closed subspace of $X$ such that $T(t) Y \subset Y$ for all $t \geq 0$, i.e., if $Y$ is $T(t)_{t \geq 0}$ invariant, then the restrictions

$$
T(t)_{\mid}:=T(t)_{\mid Y}
$$

form a $C_{0}$-semigroup $\{T(t): t \geq 0\}$, called the subspace semigroup, on the Banach space $Y$.

Lemma 5. (Proposition 2.2.3 in [6] Let $(A, D(A))$ be the generator of a $C_{0}$-semigroup $\{T(t): t \geq 0\}$ on a Banach space $X$ and assume that the restricted semigroup (subspace semigroup) $\left\{T(t)_{\mid}: t \geq 0\right\}$ is a $C_{0}$-semigroup on some $(T(t))_{t \geq 0}$-invariant Banach space $Y \rightarrow X$. Then the generator of $\left\{T(t)_{\mid}: t \geq 0\right\}$ is the part $\left(A_{\mid}, D\left(A_{\mid}\right)\right)$of $A$ in $Y$.

Lemma 6. The operator $\Delta_{\mid b D L_{2}(\Omega)}$ with $D\left(\Delta_{\mid b D L_{2}(\Omega)}\right)=\left\{u \in D(\Delta) \cap b D L_{2}(\Omega), \Delta u \in\right.$ $\left.b D L_{2}(\Omega)\right\}$ is the infinitesimal generator of an analytic semigroup of contractions on $b D L_{2}(\Omega)$.

Proof. From Theorem 7.3.6 in [11] $\Delta$ is the infinitesimal generator of an analytic semigroup of contractions on $L^{2}(\Omega)$. Then $\Delta$ is also the infinitesimal generator of an analytic semigoup of contractions on $L_{2}(\Omega)$. Let $\{T(t) \mid t \geq 0\}$ be the restriction of the analytic semigroup generated by $\Delta$ on $L_{2}(\Omega)$ to the real axis . $\{T(t) \mid t \geq 0\}$ is a $C_{0}$ semigroup of contractions by Theorem 7.2.5 and Theorem 3.1.1 in [11]. We have already noted that $b D L_{2}(\Omega)$ is a closed subspace of $L_{2}(\Omega)$ and is also a Hilbert space. We want to show that $b D L_{2}(\Omega)$ is $T(t)_{t \geq 0}$ - invariant.

For every $u \in C_{0, b}^{\infty}(\Omega), \quad \operatorname{div} \quad b u=0 \quad$ and $\lambda \in \rho(\Delta) \cap \Sigma_{\vartheta}=\{\lambda: \vartheta-\pi<\arg \lambda<$ $\pi-\vartheta,|\lambda| \geq r\}$ we have $(\lambda I-\Delta)[R(\lambda: \Delta) b u]=b u$ where $\sum_{\vartheta}$ is the same as in the proof of 
lemma 3. Since $b R(\lambda: \Delta) u=b \int_{0}^{\infty} e^{-\lambda t} T(t) u d t=\int_{0}^{\infty} e^{-\lambda t} T(t) b(x) u d t=R(\lambda: \Delta) b u$. From Lemma 3 it follows that $\operatorname{div} b R(\lambda: \Delta) u=\operatorname{div}(R(\lambda: \Delta) b u)=0$. Since $R(\lambda: \Delta)$ is bounded and so is continuous. Hence $R(\lambda, \Delta) u \in C_{0}^{\infty}$. That is to say that $C_{0, b}^{\infty}$ is $R(\lambda: \Delta)$-invariant for $\lambda \in \rho(\Delta) \cap \Sigma_{\vartheta}$. From Theorem 2.5.2 (c) in [11] it follows that $\rho(\Delta) \supset R^{+}$, and so $\rho(\Delta) \cap \Sigma_{\vartheta} \supset\{\lambda: \lambda \geq r\}$. Hence $C_{0, b}^{\infty}$ is $R(\lambda: \Delta)$-invariant for every $\lambda \geq r$. Let $u \in b D L_{2}(\Omega)$ then there exists a sequence $u_{n}$ such that $\lim _{n \rightarrow \infty} u_{n}=u$ and $u_{n} \in C_{0, b}^{\infty}$ for $n=1,2, \ldots$. Hence $R(\lambda, \Delta) u_{n} \in C_{0, b}^{\infty}$ and

$$
\lim _{n \rightarrow \infty} R(\lambda: \Delta) u_{n}=R(\lambda: \Delta) u
$$

Therefore $R(\lambda: \Delta) u \in b D L_{2}(\Omega)$ for every $\lambda \geq r$. It follows that $b D L_{2}(\Omega)$ is $R(\lambda: \Delta)$ -invariant for every $\lambda \geq r$. Now the Theorem 4.5 .1 in [11] implies that $b D L_{2}(\Omega)$ is $T(t)_{t \geq 0}-$ invariant. From Lemma 4 and Lemma 5 it follows that $\Delta_{\mid b D L_{2}(\Omega)}$ is the infinitesimal generator of the $C_{0}$ semigroup $\left\{T(t)_{\mid b D L_{2}(\Omega)}: t \geq 0\right\}$ of contractions on $b D L_{2}(\Omega)$.

We will prove that $\left\{T(t)_{\mid b D L_{2}(\Omega)}: t \geq 0\right\}$ can also be extended to an analytic semigroup on $b D L_{2}(\Omega)$. Suppose that $\lambda \in \rho(\Delta)$, i.e. there exists $R(\lambda: \Delta)$ from $L_{2}(\Omega)$ into $D(\Delta)$. Then for any $u \in b D L_{2}(\Omega) \subset L_{2}(\Omega)$, we have

$$
(\lambda I-\Delta) R(\lambda: \Delta) u=u \text { and } R(\lambda: \Delta)(\lambda I-\Delta) u=u \text {. }
$$

Thus the formula (9) becomes

$$
\left(\lambda I-\Delta_{\mid b D L_{2}(\Omega)}\right) R(\lambda: \Delta)_{\mid b D L_{2}(\Omega)} u=u \text { and } R(\lambda: \Delta)_{\mid b D L_{2}(\Omega)}\left(\lambda I-\Delta_{\mid b D L_{2}(\Omega)}\right) u=u \text {. }
$$

Hence $\left(\lambda I-\Delta_{\mid b D L_{2}(\Omega)}\right) R(\lambda: \Delta)_{\mid b D L_{2}(\Omega)}=I$ and $R(\lambda: \Delta)_{\mid b D L_{2}(\Omega)}\left(\lambda I-\Delta_{\mid b D L_{2}(\Omega)}\right)=I$. We get

$$
\left(\lambda I-\Delta_{\mid b D L_{2}(\Omega)}\right)^{-1}=R(\lambda: \Delta)_{\mid b D L_{2}(\Omega)} .
$$

From the formula (10) and Theorem 2.5.2(c) in [11] we have

$$
\rho\left(\Delta_{\mid b D L_{2}(\Omega)}\right) \supset \rho(\Delta) \supset \Sigma=\{\lambda:|\arg \lambda|\langle\pi / 2+\delta\} \cup\{0\}
$$

where $0<\delta<\pi / 2$. Thus, for $\lambda \in \Sigma, \lambda I-\Delta_{\mid b D L_{2}(\Omega)}$ is invertible. From Theorem 2.5.2(c) in 
[11] we have for $\lambda \in \Sigma, \lambda \neq 0$

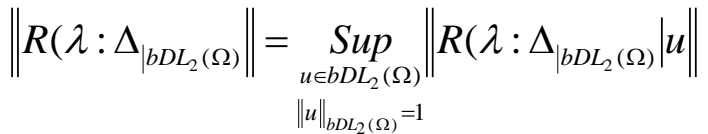

$$
\begin{aligned}
& \leq \operatorname{Sup}_{u \in L_{2}(\Omega)}\|R(\lambda: \Delta) u\|=\|R(\lambda, \Delta)\|_{B\left(L_{2}(\Omega), L_{2}(\Omega)\right)} \leq \frac{M}{|\lambda|} . \\
& \|u\|_{L_{2}(\Omega)}=1
\end{aligned}
$$

Now Theorem 2.5.2(c) in [11] implies that $\left\{T(t)_{\mid b D L_{2}(\Omega)}: \lambda \geq 0\right\}$ can also be extended to an analytic semigroup on $b D L_{2}(\Omega)$. Therefor $\Delta_{\mid b D L_{2}(\Omega)}$ is a infinitesimal generator of an analytic semigroup of contraction on $b D L_{2}(\Omega)$.We always denote $\Delta_{\mid b D L_{2}(\Omega)}$ by $\Delta$ in the follows.

Since $b D L_{2}(\Omega)=D L_{2}(\Omega)$ in the case $b$ is a constant. So we have

Corollary 1. The operator $\Delta$ is the infinitesimal generator of an analytic semigroup of contractions on $D L_{2}(\Omega)$.

Theorem 2 in [9] is similar to the above Lemma 6. Sobolevskii proved the fact that $A_{p}=$ $P \Delta$ generates an analytic semigroup on $L_{\sigma}^{2}(\Omega)=D L_{2}(\Omega)$ in [17 ] . Giga gave a different proof in [9]. Our proof using the theory of semigroups of bounded operators is more simple.

Lemma 7. Suppose that $b \in C^{\infty}(\Omega)$ then $D L_{2}(\Omega) \subseteq b D L_{2}(\Omega)$.

Proof. If $b \in C^{\infty}(\Omega)$ then $b \in W^{1,2}(\Omega), \nabla b \in\left(D L_{2}(\Omega)\right)^{\perp}$, so $(u, \nabla b)=0$ for every $u \in C_{0, \sigma}^{\infty}(\Omega) \subset D L_{2}(\Omega)$, that is

$$
\int_{\Omega}\left(\sum_{i=1}^{3} u_{i} \frac{\partial b}{\partial x_{i}}\right) d x=0 .
$$

So $\sum_{i=1}^{3} u_{i} \frac{\partial b}{\partial x_{i}}=0$ for every $u \in C_{0, \sigma}^{\infty}$. Hence

$$
\operatorname{div}[b u]=\sum_{i=1}^{3} \frac{\partial\left[b u_{i}\right]}{\partial x_{i}}=\sum_{i=1}^{3} \frac{\partial b}{\partial x_{i}} u_{i}+b \sum_{i=1}^{3} \frac{\partial u_{i}}{\partial x_{i}}=0
$$

So $u \in C_{0, b}^{\infty}(\Omega)$ and $C_{0, \sigma}^{\infty}(\Omega) \subset C_{0, b}^{\infty}(\Omega)$. Therefore

$$
D L_{2}(\Omega)=\overline{C_{0, \sigma}^{\infty}(\Omega)} \subseteq \overline{C_{0, b}^{\infty}(\Omega)}=b D L_{2}(\Omega)
$$


Suppose that $-A$ is the infinitesimal generator of an analytic semigroup $T(t)$ on a Banach space $X$. From the results of section 2.6 in [11] we can define the fraction powers $A^{\alpha}$ for $0 \leq \alpha \leq 1$ and $A^{\alpha}$ is a closed linear invertible operator with domain $D\left(A^{\alpha}\right)$ dense in $X$. $D\left(A^{\alpha}\right)$ equipped with the norm $\|x\|_{\alpha}=\left\|A^{\alpha} x\right\|$ is a Banach space denoted by $X_{\alpha}$. It is clear that $0<\alpha<\beta$ implies $X_{\alpha} \supset X_{\beta}$ and that the embedding of $X_{\beta}$ into $X_{\alpha}$ is continuous.

$$
\text { If }-A=\Delta \text { and } \quad \gamma>1 / 2 \text { then } X_{\gamma} \subset X_{\frac{1}{2}} \text { and } D\left((-\Delta)^{\gamma}\right) \subset D\left((-\Delta)^{1 / 2}\right) \subset D L_{2}(\Omega) \text {, }
$$

the embedding of $D\left((-\Delta)^{\gamma}\right)$ into $D\left((-\Delta)^{\frac{1}{2}}\right)$ is continuous. If a sequence $u_{n}(n=1,2$, $\ldots) \in D\left((-\Delta)^{\gamma}\right), \lim _{n \rightarrow \infty}\left\|u_{n}\right\|_{D L_{2}(\Omega)_{\gamma}}=0$, then $\lim _{n \rightarrow \infty}\left\|u_{n}-0\right\|_{D L_{2}(\Omega)_{\gamma}}=0$, that is $\lim _{n \rightarrow \infty} u_{n}=0$ in $D\left((-\Delta)^{\gamma}\right)$, and so $\lim _{n \rightarrow \infty} u_{n}=0$ in $D\left((-\Delta)^{\frac{1}{2}}\right)$. It follows from Proposition 1.17 in [3] that $\lim _{n \rightarrow \infty}\left\|u_{n}\right\|_{D L_{2}(\Omega)_{\frac{1}{2}}}=0$. Hence $\left\|u_{n}\right\|_{D L_{2}(\Omega)_{\gamma}} \rightarrow 0$ always implies $\left\|u_{n}\right\|_{D L_{2}(\Omega)_{\frac{1}{2}}} \rightarrow 0$. We can prove that convergence in $\left(D\left((-\Delta)^{\gamma}\right),\|\bullet\|_{D L_{2}(\Omega)_{\gamma}}\right)$ implies convergence in $\left(D\left((-\Delta)^{\frac{1}{2}}\right),\|\bullet\|_{D L_{2}(\Omega)_{1 / 2}}\right)$ and conversely, that is the norms $\|\bullet\|_{D L_{2}(\Omega)_{\gamma}}$ and $\|\bullet\|_{D L_{2}(\Omega)_{\frac{1}{2}}}$ in $D\left((-\Delta)^{\gamma}\right)$ are equivalent (see p291 Problem 8 in [7] , therefore there exists $L_{0}>0$ such that for any $u \in D\left((-\Delta)^{\gamma}\right)$

$$
\left\|(-\Delta)^{\gamma} u\right\|_{D L_{2}(\Omega)}=\|u\|_{D L_{2}(\Omega)_{\gamma}} \leq L_{0}\|u\|_{D L_{2}(\Omega)_{1 / 2}} .
$$

For $u \in D(\Delta)$ we have

$$
\|\nabla u\|_{D L_{2}(\Omega)}=\|-\nabla u\|_{D L_{2}(\Omega)}=\left\|(-\Delta)^{1 / 2} u\right\|_{D L_{2}(\Omega)}=\|u\|_{D L_{2}(\Omega) \frac{1}{2}} .
$$

In [9] Giga proved the following result. Suppose that $1<r<\infty, n \geq 2$.

Lemma 8. Let $0 \leq \delta<1 / 2\left|-n\left(1-r^{-1}\right) / 2\right|$. Then

$$
\left\|A^{-\delta} P(u, \nabla) v\right\|_{0, r} \leq M\left\|A^{\vartheta} u\right\|_{0, r}\left\|A^{\omega} v\right\|_{0, r}
$$

with some constant $M=M(\delta, \vartheta, \omega, r)$, provided $\delta+\vartheta+\omega \geq n / 2 r+1 / 2, \vartheta>0, \omega$ $>0, \omega+\delta>1 / 2$.

From the Lemma 8 and the formulas (11) (12) we see that if take $n=3, r=2$, 
$\delta=0, \vartheta=3 / 4$ and $\omega=3 / 4$, then

$$
\begin{aligned}
& \|(u \bullet \nabla) v\|_{D L_{2}(\Omega)}=\|(u \bullet \nabla) v\|_{L_{2}(\Omega)}=\|(u \bullet \nabla) v\|_{W^{0,2}(\Omega)} \\
& \leq M\left\|(-\Delta)^{\vartheta} u\right\|_{D L_{2}(\Omega)}\left\|(-\Delta)^{\omega} v\right\|_{D L_{2}(\Omega)} \\
& \leq M L_{0}^{2}\|u\|_{D L_{2}(\Omega)_{1 / 2}}\|v\|_{D L_{2}(\Omega)_{1 / 2}}
\end{aligned}
$$

with some constant $M$ for any $u, v \in D L_{2}(\Omega)$. Hence we have

Lemma 9. Suppose that $u, v \in D L_{2}(\Omega)$ are velocity fields and $(u \bullet \nabla) v \in$ $D L_{2}(\Omega)$, then

$$
\|(u \bullet \nabla) v\|_{D L_{2}(\Omega)} \leq M L_{0}^{2}\|u\|_{D L_{2}(\Omega)_{1 / 2}}\|v\|_{D L_{2}(\Omega)_{1 / 2}} .
$$

Assumption (F). Let $X=b D L_{2}(\Omega)$ and $U$ be an open subset in $R^{+} \times X_{\alpha}(0<\alpha<1)$. The function $f: U \rightarrow X$ satisfies the assumption (F) if for every $(t, u) \in U$ there is a neighborhood $V \subset U$ and constants $L \geq 0, \quad 0<\vartheta \leq 1$ such that for all $\left(t_{i}, u_{i}\right) \in V(i=1,2)$

$$
\left\|f\left(t_{1}, u_{1}\right)-f\left(t_{2}, u_{2}\right)\right\| \leq L\left(\left|t_{1}-t_{2}\right|^{\vartheta}+\left\|u_{1}-u_{2}\right\|_{\alpha}\right) .
$$

Lemma 10. ( Theorem 6.3.1 in [11]) Let $-A$ be the generator of an analytic semigroup $T(t)$ on the Banach space $X=b D L_{2}\left(R^{3}\right)$ satisfying $\|T(t)\| \leq M$ and assume that $0 \in \rho(-A)$. If, $0<\alpha<1$ and $f$ satisfies the assumption (F) then for every initial date $\left(t_{0}, u_{0}\right) \in U$ the initial value problem

$$
\left\{\begin{array}{c}
\frac{d u(t)}{d t}+A u(t)=f(t, u(t)), t \in\left(t_{0}, T\right] \\
u\left(t_{0}\right)=u_{0}
\end{array}\right.
$$

has a unique local solution $u \in C\left(\left[t_{0}, t_{1}\right): X\right) \cap C^{1}\left(\left(t_{0}, t_{1}\right): X\right)$ where $t_{1}=t_{1}\left(t_{0}, u_{0}\right)>t_{0}$.

In what follows we will need Banach lattice (see [1] ). A real vector space $G$ which is ordered by some order relation $\leq$ is called a vector lattice (or Riesz space) if any two elements $f, g \in G$ have a least upper bound, denoted by $f \vee g$, and a greatest lower bound, denoted by $f \wedge g$, and the following properties are satisfied: 
(i) If $f \leq g$, then $f+h \leq g+h$ for all $f, g, h \in G$,

(ii) If $0 \leq f$, then $0 \leq t f$ for all $f \in G$ and $0 \leq t \in R$.

A Banach lattice is a real Banach space $G$ endowed with an ordering $\leq$ such that $(G, \leq)$ is a vector lattice and the norm is a lattice norm, that is $|f| \leq|g|$ implies $\|f\| \leq\|g\|$ for $f, g \in G$, where $|f|=f \vee(-f)$ is the absolute value of $f$ and $\|\bullet\|$ is the norm in $G$. In a Banach lattice $G$ we define for $f \in G$

$$
f^{+}:=f \vee 0, \quad f^{-}:=(-f) \vee 0
$$

The absolute value of $f$ is $|f|=f^{+}+f^{-}$and $f=f^{+}-f^{-}$.

$$
0 \leq f \leq g \Rightarrow|f| \leq|g| \Rightarrow\|f\| \leq\|g\| .
$$

In what follows we will need the above formula .

In $L^{2}(\Omega)$ we define the order for $f, g \in L^{2}(\Omega)$

$$
f \leq g \Leftrightarrow f(x) \leq g(x) \text { for a.e. } x \in \Omega
$$

and

$$
(f \vee g)(x):=\max \{f(x), g(x)\}, \quad(f \wedge g)(x):=\min \{f(x), g(x)\}
$$

for a.e. $x \in \Omega$. Then $W^{1,2}(\Omega), L^{2}(\Omega), L_{2}(\Omega)$ and $D L_{2}(\Omega)$ are all Banach lattices. (see [1] p.148)

Lemma11. Suppose that $u, v \in D L_{2}(\Omega)$ are divergence free satisfying $\frac{\partial u_{i}}{\partial x_{j}}=0, \frac{\partial v_{i}}{\partial x_{j}}=0$ $(i \neq j)$. Then $(u \bullet \nabla) v, \quad(v \bullet \nabla) u \in D L_{2}(\Omega)$.

Proof. If $u, v \in D L_{2}(\Omega)$ are divergence free satisfying $\frac{\partial u_{i}}{\partial x_{j}}=0, \frac{\partial v_{i}}{\partial x_{j}}=0(i \neq j)$. From [4] and [8]we have

$$
\int_{\Omega} u \bullet \nabla h d x=0, \int_{\Omega} v \bullet \nabla h d x=0 \text { for all } h \in W^{1,2}(\Omega) .
$$

That is $\int_{\Omega} \sum_{i=1}^{3} u_{i} \frac{\partial h}{\partial x_{i}} d x=0$. Since $L_{2}(\Omega)$ is a Banach lattice and $\nabla h \in D L_{2}(\Omega)^{\perp}$ for $h \in W^{1,2}(\Omega)$, then $u=u^{+}-u^{-}, u^{+}, u^{-} \in D L_{2}(\Omega), \nabla h=(\nabla h)^{+}-(\nabla h)^{-}$. From proposition 10.8 
in [1] the lattice operations $\wedge$ and $\vee$ are continuous, we have

$$
\begin{aligned}
& (\nabla h)^{+}=\left(\begin{array}{c}
\frac{\partial h}{\partial x_{1}} \\
\frac{\partial h}{\partial x_{2}} \\
\frac{\partial h}{\partial x_{3}}
\end{array}\right)=\left(\begin{array}{c}
\left(\frac{\partial h}{\partial x_{1}}\right)^{+} \\
\left(\frac{\partial h}{\partial x_{2}}\right)^{+} \\
\left(\frac{\partial h}{\partial x_{3}}\right)^{+}
\end{array}\right)=\left(\begin{array}{c}
\left(\lim _{x_{1} \rightarrow x_{10}} \frac{h\left(x_{1}, x_{2}, x_{3}\right)-h\left(x_{10}, x_{2}, x_{3}\right)}{x_{1}-x_{10}}\right)^{+} \\
\left(\lim _{x_{2} \rightarrow x_{20}} \frac{h\left(x_{1}, x_{2}, x_{3}\right)-h\left(x_{1}, x_{20}, x_{3}\right)}{x_{2}-x_{20}}\right)^{+} \\
\left.\lim _{x_{3} \rightarrow x_{30}} \frac{h\left(x_{1}, x_{2}, x_{3}\right)-h\left(x_{1}, x_{2}, x_{30}\right)}{x_{3}-x_{30}}\right)^{+}
\end{array}\right) \\
& \left.\lim _{x_{1} \rightarrow x_{10}} \frac{h^{+}\left(x_{1}, x_{2}, x_{3}\right)-h^{+}\left(x_{10}, x_{2}, x_{3}\right)}{x_{1}-x_{10}}\right)\left(\begin{array}{l}
\frac{\partial h^{+}}{\partial x_{1}} \\
\lim _{x_{2} \rightarrow x_{20}} \frac{h^{+}\left(x_{1}, x_{2}, x_{3}\right)-h^{+}\left(x_{1}, x_{20}, x_{3}\right)}{x_{2}-x_{20}}
\end{array}\right)=\left(\begin{array}{l}
\frac{\partial h^{+}}{\partial x_{2}} \\
\frac{\partial h^{+}}{\partial x_{3}}
\end{array}\right)=\nabla\left(h^{+}\right),
\end{aligned}
$$

Similarly, $(\nabla h)^{-}=\nabla\left(h^{-}\right) . W^{1,2}(\Omega) \subset W^{0,2}(\Omega)=L_{2}(\Omega)$ are all Banach spaces. $h \in W^{1,2}(\Omega)$ implies $h^{+}, h^{-} \in W^{1,2}(\Omega), \int_{\Omega} u^{+} \bullet \nabla\left(h^{+}\right) d x=0$. Since $v$ is divergence free, $\sum_{i=1}^{3} \frac{\partial v_{i}}{\partial x_{i}}=0$, so $\frac{\partial v_{i}}{\partial x_{i}}$ are all bounded, $\left|\frac{\partial v_{i}}{\partial x_{i}}\right| \leq L \quad(\mathrm{i}=1,2,3)$ for some constant $L>0$. We have

$$
\begin{aligned}
& 0=-L \int_{\Omega} \sum_{i=1}^{3} u_{i}^{+}\left(\frac{\partial h}{\partial x_{i}}\right)^{+} d x \leq \int_{\Omega}\left(u^{+} \bullet \nabla\right) v \bullet(\nabla h)^{+} d x \\
& =\int_{\Omega} \sum_{i=1}^{3} u_{i}^{+} \frac{\partial v_{i}}{\partial x_{i}}\left(\frac{\partial h}{\partial x_{i}}\right)^{+} d x \leq L \int_{\Omega} \sum_{i=1}^{3} u_{i}^{+} \frac{\partial\left(h^{+}\right)}{\partial x_{i}} d x=0
\end{aligned}
$$

Hence $\int_{\Omega}\left(u^{+} \bullet \nabla\right) v \bullet(\nabla h)^{+} d x=0$. Similarly, $\int_{\Omega}\left(u^{+} \bullet \nabla\right) v \bullet(\nabla h)^{-} d x=0$. So we have

$$
\int_{\Omega}\left(u^{+} \bullet \nabla\right) v \bullet \nabla h d x=\int_{\Omega}(u \bullet \nabla) v \bullet\left[(\nabla h)^{+}-(\nabla h)^{-}\right] d x=0 .
$$

Similarly, $\int_{\Omega}\left(u^{-} \bullet \nabla\right) v \bullet \nabla h d x=0$. Therefore

$\int_{\Omega}(u \bullet \nabla) v \bullet \nabla h d x=\int_{\Omega}\left(\left(u^{+}-u^{-}\right) \bullet \nabla\right) v \bullet \nabla h d x=\int_{\Omega}\left(u^{+} \bullet \nabla\right) v \bullet \nabla h d x-\int_{\Omega}\left(u^{-} \bullet \nabla\right) v \bullet \nabla h d x=0$

and so $(u \bullet \nabla) v \in D L_{2}(\Omega)$. Similarly $(v \bullet \nabla) u \in D L_{2}(\Omega)$. 


\section{(3)The solutions of lake equations}

Now we study the viscous lake equations (2). In the following proof of Theorem 1 we will need valuation for nonlinear term $(u \bullet \nabla) u$. Lemma 9 gives a good valuation for $(u \bullet \nabla) u$ in $D L_{2}(\Omega)$. But we don not know that whether this valuation is established in $b D L_{2}(\Omega)$. From lemma 7 we see that if $b \in C^{\infty}(\Omega)$ then $D L_{2}(\Omega) \subseteq b D L_{2}(\Omega)$. Therefore we can consider the solution of (2) in the state space $D L_{2}(\Omega)$ and use lemma 9.

A function $u$ which is differentiable almost everywhere on $[0, T]$ such that $u^{\prime} \in$ $L^{1}\left[0, T: D L_{2}(\Omega)\right]$ is called a strong solution of the initial value problem $(2)$ if $u(0)=u_{0}$ and $u$ satisfies (2).

Let $H\left([0, T] ; D L_{2}(\Omega)_{\frac{1}{2}}\right)$ denote the space of all Hölder continuous functions $u(t)$ on $[0, T]$ with different exponents in $(0,1]$ and with smooth functions values $u(t)$ satisfying $\frac{\partial u_{i}}{\partial x_{j}}=0(i \neq j)$ in the Banach space $D L_{2}(\Omega)_{\frac{1}{2}}$. From the formula (4) we see that these functions values are all divergence free. Then from lemma 11 for any $u \in H\left([0, T] ; D L_{2}(\Omega)_{1 / 2}\right)$ and any $t_{1}, t_{2} \in[0, T],\left(u\left(t_{1}\right) \bullet \nabla\right) u\left(t_{2}\right) \in D L_{2}(\Omega) ;$ and for any $u_{1}, u_{2} \in H\left([0, T] ; D L_{2}(\Omega)_{1 / 2}\right)$ and any $t \in[0, T],\left(u_{1}(t) \bullet \nabla\right) u_{2}(t) \in D L_{2}(\Omega)$. In the following we will use these facts. The bilinear form $(v(t) \bullet \nabla) u(t)$ on $D L_{2}(\Omega)_{1 / 2}$ takes value in $D L_{2}(\Omega)$.

Let $\left.u_{k}(t, x)=\left(k_{1}, k_{2}, k_{3}\right) \quad\left(t \in[0, T], x \in \Omega, k_{i} \in R, i=1,2,3\right)\right)$. Then $u_{k} \in H([0, T]$ $\left.; D L_{2}(\Omega)_{1 / 2}\right)$ for all $k_{1}, k_{2}, k_{3} \in R$ and all $t \in[0, T]$. Suppose that $u(x)=$ $\left(u_{1}\left(x_{1}\right), u_{2}\left(x_{2}\right), u_{3}\left(x_{3}\right)\right) \in D L_{2}(\Omega)_{\frac{1}{2}}$ with smooth $u_{i}\left(x_{i}\right)(i=1,2,3)$. Let $u(t, x) \equiv u(x)$ for $t \in$ $[0, T]$. Then $u(t, x) \in H\left([0, T] ; D L_{2}(\Omega)_{1 / 2}\right)$. Hence $H\left([0, T] ; D L_{2}(\Omega)_{1 / 2}\right)$ is not empty. Consider the graph

$$
G:=\left\{(t, u(t)): t \in(0, T), \forall u \in H\left([0, T] ; D L_{2}(\Omega)_{1 / 2}\right)\right\}
$$


Take the open kernel $U=G^{0}$ of $G$ in $(0, T) \times D L_{2}(\Omega)_{\frac{1}{2}}$. Then $U$ is an open subset of $(0, T) \times D L_{2}(\Omega)_{\frac{1}{2}}$. It is clear that $G$ is not empty, and so $U$ is also not empty. $F(t, u(t))$ $=-(u \bullet \nabla) u$ is a function : $U \rightarrow D L_{2}(\Omega)$.

Theorem 1. The initial value problem (2) for viscous lake equations has a unique local strong solution if the initial value $\left(t_{0}, u_{0}\right) \in U$, i.e., $u_{0}=u\left(t_{0}\right)$ for some Hölder continuous function $u$ on $[0, T]$ with smooth function value $u(t) \in D L_{2}(\Omega)_{\frac{1}{2}}$ satisfying $\partial_{j} u_{i}=0(i \neq j)$ and $b(x) \in$ $C^{\infty}(\Omega)$

Proof. We will find that by incorporating the divergence-free condition, we can remove the pressure term from our equation. (see p. $271^{3}$ in [4], p. $234_{6}$ and p. $239_{9}$ in [12] ) In fact, from $D L_{2}(\Omega)^{\perp}=\left\{\nabla h ; h \in W^{1,2}(\Omega)\right\}$ we see that $\nabla p \in D L_{2}(\Omega)^{\perp}$ and so $P \nabla p=0$. For $u \in$ $D L_{2}(\Omega)$ we have $\Delta u \in D L_{2}(\Omega)$ because to Lemma 6 . Hence by applying $P$ to the equation (2) we have $P \Delta u=\Delta u$. It follows from $(u \bullet \nabla) u \in D L_{2}(\Omega)$ that $P(u \bullet \nabla) u=(u \bullet \nabla) u$. Therefore we can first rewrite (2) into an abstract initial value problem on $D L_{2}(\Omega)$

$$
\left\{\begin{array}{c}
\frac{d u}{d t}=\Delta u+F(t, u(t)), t \in\left(t_{0}, T\right] \\
\left.u\right|_{t=t_{0}}=u_{0}, x \in \Omega
\end{array}\right.
$$

where $F(t, u(t))=-(u \bullet \nabla) u$ is an abstract function. From corollary $1 \Delta$ is the infinitesimal generator of an analytic semigroup $T(t)$ of contraction on $D L_{2}(\Omega)$ and $\|T(t)\| \leq 1,0 \in \rho(\Delta)$.

If $u(t)$ is Hölder continuous about $t$ on $\left[t_{0}, T\right]_{\text {in }} D L_{2}(\Omega)_{\frac{1}{2}}$, then there is a constant $C$ and $0<\beta \leq 1$ such that

$$
\left\|u\left(t_{1}, x\right)-u\left(t_{2}, x\right)\right\|_{D L_{2}\left(R^{3}\right)_{1 / 2}} \leq C\left|t_{1}-t_{2}\right|^{\beta} \text { for } t_{1}, t_{2} \in\left[t_{0}, T\right]
$$

For any $\left(t_{1}, u_{1}\left(t_{1}\right)\right),\left(t_{2}, u_{2}\left(t_{2}\right)\right) \in U$ we have $\left(u_{1}\left(t_{1}\right) \bullet \nabla\right) u_{1}\left(t_{1}\right),\left(u_{2}\left(t_{1}\right) \bullet \nabla\right) u_{2}\left(t_{1}\right) \in D L_{2}(\Omega)$ and 


$$
\begin{aligned}
& \left\|\left(u_{1}\left(t_{1}\right) \bullet \nabla\right) u_{1}\left(t_{1}\right)-\left(u_{2}\left(t_{1}\right) \bullet \nabla\right) u_{2}\left(t_{1}\right)\right\|_{D L_{2}(\Omega)} \\
& \leq\left\|\left(u_{1}\left(t_{1}\right) \bullet \nabla\right) u_{1}\left(t_{1}\right)-\left(u_{1}\left(t_{1}\right) \bullet \nabla\right) u_{2}\left(t_{1}\right)\right\|_{D L_{2}(\Omega)} \\
& +\left\|\left(u_{1}\left(t_{1}\right) \bullet \nabla\right) u_{2}\left(t_{1}\right)-\left(u_{2}\left(t_{1}\right) \bullet \nabla\right) u_{2}\left(t_{1}\right)\right\|_{D L_{2}(\Omega)} \\
& =\left\|\left(u_{1}\left(t_{1}\right) \bullet \nabla\right)\left(u_{1}\left(t_{1}\right)-u_{2}\left(t_{1}\right)\right)\right\|_{D L_{2}(\Omega)} \\
& \left.+\|\left[\left(u_{1}\left(t_{1}\right)-u_{2}\left(t_{1}\right)\right) \bullet \nabla\right)\right] u_{2}\left(t_{1}\right) \|_{D L_{2}(\Omega)} \\
& \leq M L_{0}^{2}\left\|u_{1}\left(t_{1}\right)\right\|_{D L_{2}(\Omega)_{1 / 2}}\left\|\left(u_{1}\left(t_{1}\right)-u_{2}\left(t_{1}\right)\right)\right\|_{D L_{2}(\Omega)_{1 / 2}} \\
& \left.+\left\|u_{1}\left(t_{1}\right)-u_{2}\left(t_{1}\right)\right\|_{D L_{2}(\Omega)_{1} / 2}\left\|u_{2}\left(t_{2}\right)\right\|_{D L_{2}(\Omega)_{1 / 2}}\right) \\
& =M L_{0}^{2}\left(\left\|u_{1}\left(t_{1}\right)\right\|_{D L_{2}(\Omega)}+\left\|u_{2}\left(t_{2}\right)\right\|_{D L_{2}(\Omega)_{1 / 2}}\right)\left\|u_{1}\left(t_{1}\right)-u_{2}\left(t_{1}\right)\right\|_{D L_{2}(\Omega)_{1 / 2}} \\
& \leq M L_{0}^{2}\left(\left\|u_{1}\left(t_{1}\right)\right\|_{D L_{2}(\Omega)_{1 / 2}}+\left\|u_{2}\left(t_{2}\right)\right\|_{D L_{2}(\Omega)_{1 / 2}}\right) \\
& \left(\left\|u_{1}\left(t_{1}\right)-u_{2}\left(t_{2}\right)\right\|_{D L_{2}(\Omega)_{1 / 2}}+\left\|u_{2}\left(t_{2}\right)-u_{2}\left(t_{1}\right)\right\|_{D L_{2}(\Omega)_{1 / 2}}\right) \\
& \leq M L_{0}^{2}\left(\left\|u_{1}\left(t_{1}\right)\right\|_{D L_{2}(\Omega)_{1 / 2}}+\left\|u_{2}\left(t_{2}\right)\right\|_{D L_{2}(\Omega)_{1 / 2}}\right) \\
& \left(\left\|u_{1}\left(t_{1}\right)-u_{2}\left(t_{2}\right)\right\|_{D L_{2}(\Omega)_{1 / 2}}+C_{1}\left|t_{1}-t_{2}\right|_{1}^{\beta_{1}}\right) \\
& =2 M L_{0}^{2} L\left\|u_{1}\left(t_{1}\right)-u_{2}\left(t_{2}\right)\right\|_{D L_{2}(\Omega)_{1 / 2}}+\left.2 M L_{0}^{2} L C_{1}\right|_{1}-\left.t_{2}\right|^{\beta} .
\end{aligned}
$$

We used lemma 9 in the above third step . For any $u\left(t_{1}\right), u\left(t_{2}\right) \in D L_{2}(\Omega)_{1 / 2}$ we have

$$
\begin{aligned}
& \left\|\left(u\left(t_{1}\right) \bullet \nabla\right) u\left(t_{1}\right)-\left(u\left(t_{2}\right) \bullet \nabla\right) u\left(t_{2}\right)\right\|_{D L_{2}(\Omega)} \\
& \leq\left\|\left(u\left(t_{1}\right) \bullet \nabla\right) u\left(t_{1}\right)-\left(u\left(t_{1}\right) \bullet \nabla\right) u\left(t_{2}\right)\right\|_{D L_{2}(\Omega)}+\left\|\left(u\left(t_{1}\right) \bullet \nabla\right) u\left(t_{2}\right)-\left(u\left(t_{2}\right) \bullet \nabla\right) u\left(t_{2}\right)\right\|_{D L_{2}(\Omega)} \\
& \left.=\left\|\left(u\left(t_{1}\right) \bullet \nabla\right)\left(u\left(t_{1}\right)-u\left(t_{2}\right)\right)\right\|_{D L_{2}(\Omega)}+\|\left(u\left(t_{1}\right)-u\left(t_{2}\right)\right) \bullet \nabla\right] u\left(t_{2}\right) \|_{D L_{2}(\Omega)} \\
& \leq M L_{0}^{2}\left(\left\|u\left(t_{1}\right)\right\|_{D L_{2}(\Omega)_{1 / 2}}\left\|\left(u\left(t_{1}\right)-u\left(t_{2}\right)\right)\right\|_{D L_{2}(\Omega)_{1 / 2}}+\left\|u\left(t_{1}\right)-u\left(t_{2}\right)\right\|_{D L_{2}(\Omega)_{1 / 2}}\left\|u\left(t_{2}\right)\right\|_{D L_{2}(\Omega)_{1 / 2}}\right) \\
& =M L_{0}^{2}\left(\left\|u\left(t_{1}\right)\right\|_{D L_{2}(\Omega)_{1 / 2}}+\left\|u\left(t_{2}\right)\right\|_{D L_{2}(\Omega)_{1 / 2}}\right)\left\|u\left(t_{1}\right)-u\left(t_{2}\right)\right\|_{D L_{2}(\Omega)_{1 / 2}} \\
& \leq M L_{0}^{2} C\left(\left\|u\left(t_{1}\right)\right\|_{D L_{2}(\Omega)_{1 / 2}}+\left\|u\left(t_{2}\right)\right\|_{D L_{2}(\Omega)_{1 / 2}}\right)\left|t_{1}-t_{2}\right|^{\beta} .
\end{aligned}
$$

We used the Lemma 9 in the above third step and the formula (16) in fifth step.

Suppose that $\left(t_{0}, u_{0}\right) \in U$. Set

$$
V=B_{\varepsilon}\left(t_{0}, u_{0}\right)=\left\{(t, u(t)) \in U:\left|t-t_{0}\right|\left\langle\varepsilon \leq 1,\left\|u-u_{0}\right\|_{D L_{2}(\Omega) 1 / 2}\langle\varepsilon\} .\right.\right.
$$

Then for $(t, u(t)) \in V$, 


$$
\|u\|_{D L_{2}(\Omega)_{1 / 2}}=\left\|u-u_{0}+u_{0}\right\|_{D L_{2}(\Omega)_{1 / 2}} \leq\left\|u-u_{0}\right\|_{D L_{2}(\Omega)_{1 / 2}}+\left\|u_{0}\right\|_{D L_{2}(\Omega)_{1 / 2}} \leq \varepsilon+\left\|u_{0}\right\|_{D L_{2}(\Omega)_{1 / 2}} .
$$

Let $\quad L=\varepsilon+\left\|u_{0}\right\|_{D L_{2}(\Omega)_{1} / 2}, \quad L_{1}=2 M L_{0}^{2} L, \quad L_{2}=2 M L_{0}^{2} L\left(C_{1}+C\right), \quad L_{3}=\operatorname{Max}\left(L_{2}, L_{1}\right) \quad$ and $\beta_{2}=\operatorname{Min}\left(\beta, \beta_{1}\right)$, then from (16),(17) and (18) for all $\left(t_{i}, u_{i}\right) \in V(i=1,2)$ we have

$\left\|F\left(t_{1}, u_{1}\left(t_{1}\right)\right)-F\left(t_{2}, u_{2}\left(t_{2}\right)\right)\right\|_{D L_{2}(\Omega)}$

$\leq\left\|F\left(t_{1}, u_{1}\left(t_{1}\right)\right)-F\left(t_{1}, u_{2}\left(t_{1}\right)\right)\right\|_{D L_{2}(\Omega)}+\left\|F\left(t_{1}, u_{2}\left(t_{1}\right)\right)-F\left(t_{2}, u_{2}\left(t_{2}\right)\right)\right\|_{D L_{2}(\Omega)}$

$=\left\|\left(u_{1}\left(t_{1}\right) \bullet \nabla\right) u_{1}\left(t_{1}\right)-\left(u_{2}\left(t_{1}\right) \bullet \nabla\right) u_{2}\left(t_{1}\right)\right\|_{D L_{2}(\Omega)}+\left\|\left(u_{2}\left(t_{1}\right) \bullet \nabla\right) u_{2}\left(t_{1}\right)-\left(u_{2}\left(t_{2}\right) \bullet \nabla\right) u_{2}\left(t_{2}\right)\right\|_{D L_{2}(\Omega)}$

$\leq 2 M L_{0}^{2} L\left\|u_{1}\left(t_{1}\right)-u_{2}\left(t_{2}\right)\right\|_{\left.D L_{2}(\Omega)\right)_{1 / 2}}+2 M L_{0}^{2} L C_{1}\left|t_{1}-t_{2}\right|^{\beta_{1}}+2 M L_{0}^{2} L C\left|t_{1}-t_{2}\right|^{\beta}$

$\leq L_{1}\left\|u_{1}\left(t_{1}\right)-u_{2}\left(t_{2}\right)\right\|_{D L_{2}(\Omega) \frac{1}{2}}+L_{2}\left|t_{1}-t_{2}\right|^{\beta_{2}}$

$\leq L_{3}\left(\left|t_{1}-t_{2}\right|^{\beta_{2}}+\left\|u_{1}\left(t_{1}\right)-u_{2}\left(t_{2}\right)\right\|_{\left.D L_{2}(\Omega)\right)_{1 / 2}}\right)$.

Hence $F(t, u(t))$ satisfies the assumption $(F)$, then by lemma 10 for every initial data $\left(t_{0}, u_{0}\right) \in U$ the initial value problem (15) has a unique local solution

$$
u \in C\left(\left[t_{0}, t^{\prime}\right): D L_{2}(\Omega)\right) \cap C^{1}\left(\left(t_{0}, t^{\prime}\right): D L_{2}(\Omega)\right)
$$

where $t^{\prime}=t^{\prime}\left(u_{0}\right)$. Since $b \in C^{\infty}(\Omega)(19)$ and lemma 7 imply

$$
u \in C\left(\left[t_{0}, t^{\prime}\right): b D L_{2}(\Omega)\right) \cap C^{1}\left(\left(t_{0}, t^{\prime}\right): b D L_{2}(\Omega)\right) .
$$

Changing the value of $u$ on $\partial \Omega$ to zero we get a unique local strong solution for (2).

Using a similar induction way as Theorem 3.9 in [9] or as Theorem 5.1 in [15] we can prove that the solution $u(t, x) \in\left(C^{\infty}\left(\left[t_{0}, t^{\prime}\right) \times \Omega\right)\right)^{3}$. We can also prove directly that $u(t, x)$ is smooth. In fact, the solution (19) of (15) is also the solution of (6). The Theorem 3.4 in [9] mean that as long as the solution of (6) exists, this solution is smooth. From Theorem 3.4 in [9] we have the solution $u(t, x) \in\left(C^{\infty}\left(\left[t_{0}, t^{\prime}\right) \times \Omega\right)\right)^{3}$. Substituting $u(t, x)$ into (2) we get the solution $p(t, x)$. We also have $p(t, x) \in C^{\infty}\left(\left[t_{0}, t^{\prime}\right) \times \Omega\right)$. Since $u(t, x) \in\left(C^{\infty}\left(\left[t_{0}, t^{\prime}\right) \times \Omega\right)\right)^{3}$. It follows from $u \in b D L_{2}(\Omega)$ and lemma 1 that div $b u=0$. So the solution $u(t, x)$ is divergence-free. Hence $u=u(t, x), p(t, x)$ is the unique local strong solution of the initial value problem (2) for viscous lake equations.

The Theorem 1 has the following corollary. 
Corollary 2. The Navier-Stokes initial value problem (3) has a unique local strong solution if the initial value $\left(t_{0}, u_{0}\right) \in U$.

\section{(3) Drift homogenization}

A composite is a material containing two or more finely mixed components. Composite materials are widely used nowadays in any kind of industries. How to determine the properties of a composite material, for example thermal, electrical or linear elastic properties of materials ? It can be solved by the homogenization of a set of partial differential equations. The homogenization theory allows to describe the asymptotic behaviour as $\varepsilon \rightarrow 0$ of partial differential equations of many types. A classical problem is the elliptic Dirichlet problem

$$
\left\{\begin{array}{c}
-\operatorname{div}\left(A^{\varepsilon} \nabla u^{\varepsilon}\right)=f, x \in \Omega \\
u^{\varepsilon}=0, x \in \partial \Omega
\end{array}\right.
$$

where $f$ is given in $H^{-1}(\Omega)$ and the matrix $A^{\varepsilon}$ is the $Y$-periodic matrix.

The prescription of traditional Chinese medicine consists of a variety of Chinese herbal medicines. Its efficacy can be obtained from the homogenization of a group of partial differential equations controlled by each drug. The actual speed of a ship can be obtained from the homogenization of a set of Lake equations disturbed by the ship's dynamic speed ( drift) $v_{\varepsilon}$. The homogenization of PDEs is completed by the following three steps:

1. Fist Construct a set of partial differential equations satisfied by objective functions $u_{\varepsilon}(\varepsilon \rightarrow 0)$ and prove the existence of solutions $u_{\varepsilon}$; One of the ways to solve these equations is to change the equations into a variational formulation, that is multiplying the equation by a arbitrary(test function) $v \in H_{0}^{1}(\Omega)$, then by integrating by parts. And then use lax-Milgram theorem to prove the existence of solutions. But in this paper we will use the method of semigroups .

2. Prove that $u_{\varepsilon}$ strongly or weakly converge to $u$ in some state space when $\varepsilon \rightarrow 0$ and $u$ is the homogenization value;

3. Find the equation satisfied by $u$. Solve this equation and get $u$.

Now we discuss the lake equation in a bounded domain $\Omega$ of $R^{3}$, perturbed by oscillating term $\left(v_{\varepsilon} \bullet \nabla\right) u_{\varepsilon}$, i.e.

$$
\left\{\begin{array}{c}
\partial_{t} u_{\varepsilon}+\left(u_{\varepsilon} \bullet \nabla\right) u_{\varepsilon}+\left(v_{\varepsilon} \bullet \nabla\right) u_{\varepsilon}-\Delta u_{\varepsilon}+\nabla p_{\varepsilon}=f_{\varepsilon}, x \in \Omega, t \in\left(t_{0}, T\right] \\
\operatorname{div}\left(b u_{\varepsilon}\right)=0, x \in \Omega \\
\left.u_{\varepsilon}\right|_{t=t_{0}}=u_{\varepsilon 0,} x \in \Omega \\
u_{\varepsilon}(t, x)=0, x \in \partial \Omega
\end{array}\right.
$$


where the oscillations are produced by the sequence of vector-value functions $v_{\varepsilon}$ which strongly converges to some $v$ in $b D L_{2}(\Omega)_{1 / 2}$ when $\varepsilon \rightarrow 0$. Here $v$ is the scheduled ship speed and $v_{\varepsilon}$ is the actual approximate speed, $\lim _{\varepsilon \rightarrow 0} v_{\varepsilon}(t)=v(t)$ in $b D L_{2}(\Omega)_{1 / 2}$ for each $t \in\left[t_{0}, T\right]$. In what follows all $u_{\varepsilon}, v_{\varepsilon}$ are velocity fields.

Theorem 2. Suppose that the smooth initial value $\left(t_{0}, u_{\varepsilon 0}\right) \in U$ for each $\varepsilon>0, b(x) \in$ $C^{\infty}(\Omega), v_{\varepsilon}$ and $f_{\varepsilon}(t, x)$ are Hölder continuous in $\left[t_{0}, T\right]$ with exponent $\beta$ and the values in $D L_{2}(\Omega),\left\|v_{\varepsilon}\right\|_{D L_{2}(\Omega)_{1 / 2}} \leq L^{0}$ and $v_{\varepsilon}$ are smooth and satisfy $\frac{\partial v_{\varepsilon i}}{\partial x_{j}}=0(i \neq j)$. Then the initial value problem (20) for viscous lake equations have local smooth strong solutions .

Proof. The proof is similar to Theorem 1. First we rewrite (20) into an abstract initial value problem on the state space $D L_{2}(\Omega)_{\frac{1}{2}}$.

$$
\left\{\begin{array}{c}
\frac{d u_{\varepsilon}}{d t}=\Delta u_{\varepsilon}+F\left(t, u_{\varepsilon}(t)\right), t \in\left(t_{0}, T\right] \\
\left.u_{\varepsilon}\right|_{t=t_{0}}=u_{\varepsilon 0}, x \in \Omega
\end{array}\right.
$$

where $F\left(t, u_{\varepsilon}(t)\right)=-\left(u_{\varepsilon}(t) \bullet \nabla\right) u_{\varepsilon}(t)-\left(v_{\varepsilon}(t) \bullet \nabla\right) u_{\varepsilon}(t)+f_{\varepsilon}$ where $v_{\varepsilon}$ is fixed for $\varepsilon>0$. Let

$$
G:=\left\{(t, u(t)): t \in(0, T), \forall u \in H\left([0, T] ; D L_{2}(\Omega)_{1 / 2}\right)\right\}
$$

Take the open kernel $U=G^{0}$ of $G$ in $(0, T) \times D L_{2}(\Omega)_{\frac{1}{2}}$. Then $U$ is an open subset of $[0, T] \times D L_{2}(\Omega)_{\frac{1}{2}}$. The bilinear form $(v \bullet \nabla) u$ on $H^{0}$ takes value in $D L_{2}(\Omega)$. That is, $F\left(t, u_{\varepsilon}(t)\right)=-\left(u_{\varepsilon} \bullet \nabla\right) u_{\varepsilon}+\left(v_{\varepsilon} \bullet \nabla\right) u_{\varepsilon}$ is a function : $U \rightarrow D L_{2}(\Omega)$

Suppose that $\left(t_{0}, u_{\varepsilon 0}\right) \in U$. Set

$$
V=B_{\varepsilon}\left(t_{0}, u_{\varepsilon 0}\right)=\left\{(t, u) \in U:|t|<\varepsilon \leq 1,\left\|u-u_{\varepsilon 0}\right\|_{D L_{2}(\Omega)_{1 / 2}}<\varepsilon\right\} .
$$

Let $L=\varepsilon+\left\|u_{0}\right\|_{D L_{2}(\Omega)_{\frac{1}{2}}}, L_{1}=2 M L_{0}^{2} L$. Similarly to Theorem 1. from (16),(17) and (18) for any $\left(t_{i}, u_{\varepsilon i}\right) \in V(i=1,2)$ we have 


$$
\begin{aligned}
& \left\|F\left(t_{1}, u_{\varepsilon 1}\left(t_{1}\right)\right)-F\left(t_{2}, u_{\varepsilon 2}\left(t_{2}\right)\right)\right\|_{D L_{2}(\Omega)} \\
& \leq\left\|\left(u_{\varepsilon 1}\left(t_{1}\right) \bullet \nabla\right) u_{\varepsilon 1}\left(t_{1}\right)-\left(u_{\varepsilon 2}\left(t_{1}\right) \bullet \nabla\right) u_{\varepsilon 2}\left(t_{1}\right)\right\|_{D L_{2}(\Omega)}+\left\|\left(u_{\varepsilon 2}\left(t_{1}\right) \bullet \nabla\right) u_{\varepsilon 2}\left(t_{1}\right)-\left(u_{\varepsilon 2}\left(t_{2}\right) \bullet \nabla\right) u_{\varepsilon 2}\left(t_{2}\right)\right\|_{D L_{2}(\Omega)} \\
& +\left\|\left(v_{\varepsilon}\left(t_{1}\right) \bullet \nabla\right) u_{\varepsilon 1}\left(t_{1}\right)-\left(v_{\varepsilon}\left(t_{1}\right) \bullet \nabla\right) u_{\varepsilon 1}\left(t_{2}\right)\right\|_{D L_{2}(\Omega)}+\left\|\left(v_{\varepsilon}\left(t_{1}\right) \bullet \nabla\right) u_{\varepsilon 1}\left(t_{2}\right)-\left(v_{\varepsilon}\left(t_{2}\right) \bullet \nabla\right) u_{\varepsilon 1}\left(t_{2}\right)\right\|_{D L_{2}(\Omega)} \\
& +\left\|\left(v_{\varepsilon}\left(t_{2}\right) \bullet \nabla\right) u_{\varepsilon 1}\left(t_{2}\right)-\left(v_{\varepsilon}\left(t_{2}\right) \bullet \nabla\right) u_{\varepsilon 2}\left(t_{2}\right)\right\|_{D L_{2}(\Omega)}+\left\|f_{\varepsilon}\left(t_{1}\right)-f_{\varepsilon}\left(t_{2}\right)\right\|_{D L_{2}(\Omega)} \\
& \leq 2 M L_{0}^{2} L\left\|u_{\varepsilon 1}\left(t_{1}\right)-u_{\varepsilon 2}\left(t_{2}\right)\right\|_{D L_{2}(\Omega)_{1 / 2}}+4 M L_{0}^{2} L C_{0}\left|t_{1}-t_{2}\right|^{\beta} \\
& +\|\left(v_{\varepsilon}\left(t_{1}\right) \bullet \nabla\right)\left(( u _ { \varepsilon 1 } ( t _ { 1 } ) - u _ { \varepsilon 1 } ( t _ { 2 } ) ) \| _ { D L _ { 2 } ( \Omega ) } + \| \left[\left(v_{\varepsilon}\left(t_{1}\right)-\left(v_{\varepsilon}\left(t_{2}\right)\right) \bullet\right] u_{\varepsilon 1}\left(t_{2}\right) \|_{D L_{2}(\Omega)}\right.\right. \\
& +\left\|\left(v_{\varepsilon}\left(t_{2}\right) \bullet \nabla\right)\left(u_{\varepsilon 1}\left(t_{2}\right)-u_{\varepsilon 2}\left(t_{2}\right)\right)\right\|_{D L_{2}(\Omega)}+\left\|f_{\varepsilon}\left(t_{1}\right)-f_{\varepsilon}\left(t_{2}\right)\right\|_{D L_{2}(\Omega)} \\
& \leq 2 M L_{0}^{2} L\left\|u_{\varepsilon 1}\left(t_{1}\right)-u_{\varepsilon 2}\left(t_{2}\right)\right\|_{\left.D L_{2}(\Omega)\right)_{1 / 2}}+4 M L_{0}^{2} L C_{0}\left|t_{1}-t_{2}\right|^{\beta} \\
& +M L_{0}^{2}\left\|v_{\varepsilon}\left(t_{1}\right)\right\|_{D L_{2}(\Omega)_{1 / 2}}\left\|u_{\varepsilon 1}\left(t_{1}\right)-u_{\varepsilon 1}\left(t_{2}\right)\right\|_{D L_{2}(\Omega)_{1 / 2}}+M L_{0}^{2}\left\|v_{\varepsilon}\left(t_{1}\right)-v_{\varepsilon}\left(t_{2}\right)\right\|_{D L_{2}(\Omega)_{1} / 2}\left\|u_{\varepsilon 1}\left(t_{2}\right)\right\|_{D L_{2}(\Omega)_{1 / 2}} \\
& +M L_{0}^{2}\left\|v_{\varepsilon}\left(t_{2}\right)\right\|_{D L_{2}(\Omega)_{1 / 2}}\left(\left\|u_{\varepsilon 1}\left(t_{2}\right)-u_{\varepsilon 1}\left(t_{1}\right)\right\|_{D L_{2}(\Omega)_{1 / 2}}+\left\|u_{\varepsilon 1}\left(t_{1}\right)-u_{\varepsilon 2}\left(t_{2}\right)\right\|_{D L_{2}(\Omega)_{1 / 2}}\right)+\left\|f_{\varepsilon}\left(t_{1}\right)-f_{\varepsilon}\left(t_{2}\right)\right\|_{D L_{2}(\Omega)} \\
& \leq 2 M L_{0}^{2} L\left\|u_{\varepsilon 1}\left(t_{1}\right)-u_{\varepsilon 2}\left(t_{2}\right)\right\|_{D L_{2}(\Omega)_{1 / 2}}+4 M L_{0}^{2} L C_{0}\left|t_{1}-t_{1}\right|^{\beta} \\
& +M L_{0}^{2} L^{0} C_{1}\left|t_{1}-t_{2}\right|^{\beta}+M L_{0}^{2} L C_{2}\left|t_{1}-t_{2}\right|^{\beta}+M L_{0}^{2} L^{0}\left(C_{1}\left|t_{1}-t_{2}\right|^{\beta}+\left\|u_{\varepsilon 1}\left(t_{1}\right)-u_{\varepsilon 2}\left(t_{2}\right)\right\|_{D L_{2}(\Omega)_{1 / 2}}\right) \\
& +C_{3}\left|t_{1}-t_{2}\right|^{\beta} \\
& \leq\left(2 M L_{0}^{2} L+M L_{0}^{2} L^{0}\right)\left\|u_{\varepsilon 1}\left(t_{1}\right)-u_{\varepsilon 2}\left(t_{2}\right)\right\|_{\left.D L_{2}(\Omega)\right)_{1 / 2}}+C_{4}\left|t_{1}-t_{2}\right|^{\beta} \\
& \leq L_{1}\left(\left|t_{1}-t_{2}\right|^{\beta}+\left\|u_{1}\left(t_{1}\right)-u_{2}\left(t_{2}\right)\right\|_{D L_{2}(\Omega) \frac{1}{2}}\right), \\
& \text { where } C_{4}=\operatorname{Max}\left(4 M L_{0}^{2} L C_{0}, M L_{0}^{2} L^{0} C_{1}, M L_{0}^{2} L C_{2}, M L_{0}^{2} L^{0} C_{1}, C_{3}\right), L_{1}=\operatorname{Max}\left(M L_{0}^{2} L+C_{4}\right) \text {. }
\end{aligned}
$$$$
\text { Hence } F(t, u(t)) \text { satisfies the assumption }(F) \text {, then by lemma } 10 \text { for the initial data }
$$$$
\left(t_{0}, u_{0}\right) \in U \text { the initial value problem (21) has a unique smooth local strong solution }
$$$$
u_{\varepsilon} \in C\left(\left[t_{0}, t_{1}\right): D L_{2}(\Omega)\right) \cap C^{1}\left(\left(t_{0}, t_{1}\right): D L_{2}(\Omega)\right)
$$

Since $b \in C^{\infty}(\Omega)(22)$ and lemma 7 imply

$$
u_{\varepsilon} \in C\left(\left[t_{0}, t_{1}\right): b D L_{2}(\Omega)\right) \cap C^{1}\left(\left(t_{0}, t_{1}\right): b D L_{2}(\Omega)\right)
$$

Similarly to Theorem 1 we get smooth strong solutions $u_{\varepsilon}$ and $p_{\varepsilon}$ for $\varepsilon>0$. $\square$

Theorem 3. Suppose that $\lim _{\varepsilon \rightarrow 0} v_{\varepsilon}(t)=v(t)$ in $b D L_{2}(\Omega)_{\frac{1}{2}}$ for each $t \in\left[t_{0}, T\right]$. Then there exists $u(t) \in b D L_{2}(\Omega)_{\frac{1}{2}}$ such that $\lim _{\varepsilon \rightarrow 0} u_{\varepsilon}(t)=u(t)$ in $b D L_{2}(\Omega)_{\frac{1}{2}}$ for each $t \in\left[t_{0}, t_{1}\right]$. 
Proof. For each fixed $\varepsilon>0$ let $y_{\varepsilon}(t)$ be the fixed point in the proof of Theorem 6.3 .1 in [11]. Then

So $\quad \lim _{\varepsilon \rightarrow 0}\left((-\Delta)^{-\frac{1}{2}} y_{\varepsilon} \bullet \nabla\right)(-\Delta)^{-\frac{1}{2}} y_{\varepsilon}=\left((-\Delta)^{-\frac{1}{2}} \lim _{\varepsilon \rightarrow 0} y_{\varepsilon} \bullet \nabla\right)(-\Delta)^{-\frac{1}{2}} \lim _{\varepsilon \rightarrow 0} y_{\varepsilon}$.

$$
\begin{aligned}
& \lim _{\varepsilon \rightarrow 0}\left[\left((-\Delta)^{-\frac{1}{2}} y_{\varepsilon} \bullet \nabla\right)(-\Delta)^{-\frac{1}{2}} y_{\varepsilon}\right]=\lim _{\varepsilon \rightarrow 0}\left(\begin{array}{l}
\sum_{i=1}^{3}(-\Delta)^{-\frac{1}{2}} y_{\varepsilon i} \frac{\partial(-\Delta)^{-\frac{1}{2}} y_{\varepsilon 1}}{\partial x_{i}} \\
\sum_{i=1}^{3}(-\Delta)^{-\frac{1}{2}} y_{\varepsilon i} \frac{\partial(-\Delta)^{-\frac{1}{2}} y_{\varepsilon 2}}{\partial x_{i}} \\
\sum_{i=1}^{3}(-\Delta)^{-\frac{1}{2}} y_{\varepsilon i} \frac{\partial(-\Delta)^{-\frac{1}{2}} y_{\varepsilon 3}}{\partial x_{i}}
\end{array}\right) \\
& =\left(\begin{array}{l}
\sum_{i=1}^{3}(-\Delta)^{-\frac{1}{2}} \lim _{\varepsilon \rightarrow 0} y_{\varepsilon i}(-\Delta)^{-\frac{1}{2}} \frac{\partial\left(\lim _{\varepsilon \rightarrow 0} y_{\varepsilon 1}\right)}{\partial x_{i}} \\
\sum_{i=1}^{2}(-\Delta)^{-\frac{1}{2}} \lim _{\varepsilon \rightarrow 0} y_{\varepsilon i}(-\Delta)^{-\frac{1}{2}} \frac{\partial\left(\lim _{\varepsilon \rightarrow 0} y_{\varepsilon 2}\right)}{\partial x_{i}} \\
\sum_{i=1}^{3}(-\Delta)^{-\frac{1}{2}} \lim _{i \rightarrow 0} y_{\varepsilon i}(-\Delta)^{-\frac{1}{2}} \frac{\partial\left(\lim _{\varepsilon \rightarrow 0} y_{\varepsilon 3}\right.}{\partial x_{i}}
\end{array}\right),
\end{aligned}
$$

Similarly,

$$
\lim _{\varepsilon \rightarrow 0}\left(v_{\varepsilon} \bullet \nabla\right) v_{\varepsilon}=\left(\lim _{\varepsilon \rightarrow 0} v_{\varepsilon} \bullet \nabla\right) \lim _{\varepsilon \rightarrow 0} v_{\varepsilon}
$$

From (3.10)in [11] we have

$$
\begin{aligned}
& y_{\varepsilon}(t)=T(t)(-\Delta)^{\frac{1}{2}} u_{0}+\int_{t_{0}}^{t}(-\Delta)^{\frac{1}{2}} T(t-s)\left[\left((-\Delta)^{-\frac{1}{2}} y_{\varepsilon} \bullet \nabla\right)(-\Delta)^{-\frac{1}{2}} y_{\varepsilon}+\left(v_{\varepsilon} \bullet \nabla\right) v_{\varepsilon}\right] d s \\
& y_{\varepsilon}(t)-T(t)(-\Delta)^{\frac{1}{2}} u_{0}-\int_{t_{0}}^{t}(-\Delta)^{\frac{1}{2}} T(t-s)\left((-\Delta)^{-\frac{1}{2}} y_{\varepsilon}(s) \bullet \nabla\right)(-\Delta)^{-\frac{1}{2}} y_{\varepsilon}(s) d s \\
& =\int_{t_{0}}^{t}(-\Delta)^{\frac{1}{2}} T(t-s)\left(v_{\varepsilon}(s) \bullet \nabla\right) v_{\varepsilon}(s) d s
\end{aligned}
$$

Since $(-\Delta)^{\frac{1}{2}} T(t-s)$ is bounded by Theorem 2.16.13(c) in [11], hence the below of (23) has a

limit when $\varepsilon \rightarrow 0$ by

Theorem 7.16 in [13].

$$
\begin{aligned}
& -\lim _{\varepsilon \rightarrow 0} \int_{t_{0}}^{t}(-\Delta)^{\frac{1}{2}} T(t-s)\left(v_{\varepsilon}(s) \bullet \nabla\right) v_{\varepsilon}(s) d s \\
& =-\int_{t_{0}}^{t}(-\Delta)^{\frac{1}{2}} T(t-s)\left(\lim _{\varepsilon \rightarrow 0} v_{\varepsilon}(s) \bullet \nabla\right) \lim _{\varepsilon \rightarrow 0} v_{\varepsilon}(s) d s . \\
& =-\int_{t_{0}}^{t}(-\Delta)^{\frac{1}{2}} T(t-s)(v(s) \bullet \nabla) v(s) d s
\end{aligned}
$$

Therefor the above of (23) also has a limit and so there exists $\lim _{\varepsilon \rightarrow 0} y_{\varepsilon}(t)=y(t)$. Otherwise, $y_{\varepsilon}(t),(-\Delta)^{-\frac{1}{2}} y_{\varepsilon}(t)$ and $\int_{t_{0}}^{t}(-\Delta)^{\frac{1}{2}} T(t-s)\left((-\Delta)^{-\frac{1}{2}} y_{\varepsilon}(s) \bullet \nabla\right)(-\Delta)^{-\frac{1}{2}} y_{\varepsilon}(s) d s$ are all unbounded or oscillatory, and they cannot be offset, inducing the above of (23) unbounded or oscillating, a contradiction.

Since $u_{\varepsilon}(t)=(-\Delta)^{-\frac{1}{2}} y_{\varepsilon}(t)$, so there exists limit 


$$
\lim _{\varepsilon \rightarrow 0} u_{\varepsilon}(t)=\lim _{\varepsilon \rightarrow 0}(-\Delta)^{-\frac{1}{2}} y_{\varepsilon}(t)=(-\Delta)^{-\frac{1}{2}} \lim _{\varepsilon \rightarrow 0} y_{\varepsilon}(t)=(-\Delta)^{-\frac{1}{2}} y(t)=u(t)
$$

for $t \in\left[t_{0}, t_{1}\right]$

Theorem 4. Assume that $\lim _{\varepsilon \rightarrow 0} v_{\varepsilon}=v, \lim _{\varepsilon \rightarrow 0} f_{\varepsilon}=f$ in $b D L_{2}(\Omega)$ and there exists $p \in$ $C^{\infty}\left(\left[t_{0}, T\right] \times \Omega\right)$ such that $\lim _{\varepsilon \rightarrow 0} \nabla p_{\varepsilon}=\nabla p$, then $u, p$ satisfies

$$
\left\{\begin{array}{c}
\partial_{t} u+(u \bullet \nabla) u+(v \bullet \nabla) u-\Delta u+\nabla p=f \\
\operatorname{div}(b u)=0, x \in \Omega \\
\left.u\right|_{t=t_{0}}=u_{0}, x \in \Omega \\
u(t, x)=0, x \in \partial \Omega
\end{array} .\right.
$$

Proof. From theorem $3 \lim _{\varepsilon \rightarrow 0} v_{\varepsilon}(t)=v(t)$ implies $\lim _{\varepsilon \rightarrow 0} u_{\varepsilon}(t)=u(t)$, i.e. $\lim _{\varepsilon \rightarrow 0}\left\|u_{\varepsilon}(t)-u(t)\right\|_{b D L_{2}(\Omega)}=$ 0 for each $t \in\left[t_{0}, T\right]$. This strong convergence is uniform for $x \in \Omega$. Now by using similar Theorem 7.11 in [13] we have

(1) $\lim _{\varepsilon \rightarrow 0} \frac{\partial u_{\varepsilon}}{\partial t}=\lim _{\varepsilon \rightarrow 0} \lim _{t \rightarrow t^{\prime}} \frac{u_{\varepsilon}(t)-u_{\varepsilon}\left(t^{\prime}\right)}{t-t^{\prime}}=\lim _{t \rightarrow t^{\prime}} \lim _{\varepsilon \rightarrow 0} \frac{u_{\varepsilon}(t)-u_{\varepsilon}\left(t^{\prime}\right)}{t-t^{\prime}}=\lim _{t \rightarrow t^{\prime}} \frac{u(t)-u\left(t^{\prime}\right)}{t-t^{\prime}}=\frac{\partial u}{\partial t}$.

(2) Let $u_{\varepsilon}=\left(u_{\varepsilon 1}, u_{\varepsilon 2}, u_{\varepsilon 3}\right), u=\left(u_{1}, u_{2}, u_{3}\right)$.

$\lim _{\varepsilon \rightarrow 0} \frac{\partial u_{\varepsilon i}}{\partial x_{i}}=\lim _{\varepsilon \rightarrow 0} \lim _{x_{i} \rightarrow x_{i 0}} \frac{u_{\varepsilon i}\left(x_{i}\right)-u_{\varepsilon i}\left(x_{i 0}\right)}{x_{i}-x_{i 0}}=\lim _{x_{i} \rightarrow x_{i 0}} \lim _{\varepsilon \rightarrow 0} \frac{u_{\varepsilon i}\left(x_{i}\right)-u_{\varepsilon i}\left(x_{i 0}\right)}{x_{i}-x_{i 0}}=\lim _{x_{i} \rightarrow x_{i 0}} \frac{u_{i}\left(x_{i}\right)-u_{i}\left(x_{i 0}\right)}{x_{i}-x_{i 0}}=\frac{\partial u_{i}}{\partial x_{i}}$

for $i=1,2,3$. This is to say that $\lim _{\varepsilon \rightarrow 0} u_{\varepsilon}=u$ implies $\lim _{\varepsilon \rightarrow 0} \frac{\partial u_{\varepsilon i}}{\partial x_{i}}=\frac{\partial u_{i}}{\partial x_{i}}$ for $i=1,2,3$.

$$
\begin{aligned}
& \lim _{\varepsilon \rightarrow 0} \frac{\partial^{2} u_{\varepsilon i}}{\partial x_{i}^{2}}=\lim _{\varepsilon \rightarrow 0} \frac{\partial}{\partial x_{i}} \frac{\partial u_{\varepsilon i}}{\partial x_{i}}=\lim _{\varepsilon \rightarrow 0} \lim _{x_{i} \rightarrow x_{i 0}} \frac{\frac{\partial u_{i j}}{\partial x_{i}}\left(x_{i}\right)-\frac{\partial u_{\varepsilon i}}{\partial x_{i}}\left(x_{i 0}\right)}{x_{i}-x_{i 0}} \\
& =\lim _{x_{i} \rightarrow x_{i 0}} \lim _{\varepsilon \rightarrow 0} \frac{\frac{\partial u_{i i}}{\partial x_{i}}\left(x_{i}\right)-\frac{\partial u_{i i}}{\partial x_{i}}\left(x_{i 0}\right)}{x_{i}-x_{i 0}}=\lim _{x_{i}-x_{i 0}} \frac{\frac{\partial u_{i}}{\partial x_{i}}\left(x_{i}\right)-\frac{\partial u_{i}}{\partial x_{i}}\left(x_{i 0}\right)}{x_{i}-x_{i 0}}=\frac{\partial^{2} u_{i}}{\partial x_{i}^{2}}
\end{aligned}
$$

for $i=1,2,3$. This to say that $\lim _{\varepsilon \rightarrow 0} u_{\varepsilon}=u$ implies $\lim _{\varepsilon \rightarrow 0} \frac{\partial^{2} u_{\varepsilon i}}{\partial x_{i}^{2}}=\frac{\partial^{2} u_{i}}{\partial x_{i}^{2}}$ for $i=1,2,3$. $\lim _{\varepsilon \rightarrow 0} \Delta u_{\varepsilon i}=$ $\lim _{\varepsilon \rightarrow 0} \sum_{j=1}^{3} \frac{\partial^{2} u_{\varepsilon i}}{\partial x_{j}{ }^{2}}=\sum_{j=1}^{3} \frac{\partial^{2} u_{i}}{\partial x_{j}{ }^{2}}=\Delta u_{i}$ for $i=1,2,3$. So $\lim _{\varepsilon \rightarrow 0} \Delta u_{\varepsilon}=\Delta u$.

(3) For $i=1,2,3$ 


$$
\begin{aligned}
& \lim _{\varepsilon \rightarrow 0} u_{\varepsilon i} \frac{\partial u_{\varepsilon i}}{\partial x_{i}}=\lim _{\varepsilon \rightarrow 0}\left[u_{\varepsilon i} \lim _{x_{i} \rightarrow x_{i 0}} \frac{u_{\varepsilon i}\left(x_{i}\right)-u_{\varepsilon i}\left(x_{i 0}\right)}{x_{i}-x_{i 0}}\right]=\lim _{\varepsilon \rightarrow 0} u_{\varepsilon i} \lim _{\varepsilon \rightarrow 0} \lim _{x_{i} \rightarrow x_{i 0}} \frac{u_{\varepsilon i}\left(x_{i}\right)-u_{\varepsilon i}\left(x_{i 0}\right)}{x_{i}-x_{i 0}} \\
& =u_{i} \lim _{x_{i} \rightarrow x_{i 0}} \lim _{\varepsilon \rightarrow 0} \frac{u_{\varepsilon i}\left(x_{i}\right)-u_{\varepsilon i}\left(x_{i 0}\right)}{x_{i}-x_{i 0}}=u_{i} \lim _{x_{i} \rightarrow x_{i 0}} \frac{u_{i}\left(x_{i}\right)-u_{i}\left(x_{i 0}\right)}{x_{i}-x_{i 0}}=u_{i} \frac{\partial u_{i}}{\partial x_{i}} .
\end{aligned}
$$

And so

$$
\lim _{\varepsilon \rightarrow 0}\left(u_{\varepsilon} \bullet \nabla\right) u_{\varepsilon}=\lim _{\varepsilon \rightarrow 0}\left(\sum_{i=1}^{3} u_{\varepsilon i} \frac{\partial}{\partial x_{i}}\right)\left(\begin{array}{l}
u_{\varepsilon 1} \\
u_{\varepsilon 2} \\
u_{\varepsilon 3}
\end{array}\right)=\lim _{\varepsilon \rightarrow 0}\left(\begin{array}{l}
\sum_{i=1}^{3} u_{\varepsilon i} \frac{\partial u_{\varepsilon 1}}{\partial x_{i}} \\
\sum_{i=1}^{3} u_{\varepsilon i} \frac{\partial u_{\varepsilon 2}}{\partial x_{i}} \\
\sum_{i=1}^{3} u_{\varepsilon i} \frac{\partial u_{\varepsilon 3}}{\partial x_{i}}
\end{array}\right)=\left(\begin{array}{l}
\sum_{i=1}^{2} u_{i} \frac{\partial u_{1}}{\partial x_{i}} \\
\sum_{i=1}^{3} u_{i} \frac{\partial u_{2}}{\partial x_{i}} \\
\sum_{i=1}^{3} u_{i} \frac{\partial u_{3}}{\partial x_{i}}
\end{array}\right)=(u \bullet \nabla) u .
$$

Similarly, $\lim _{\varepsilon \rightarrow 0}\left(v_{\varepsilon} \bullet \nabla\right) u_{\varepsilon}=(v \bullet \nabla) u$.

(4) Now from (1),(2),(3) and the equations (20) we take limit and get

$$
\partial_{t} u+(u \bullet \nabla) u+(v \bullet \nabla) u-\Delta u+\nabla p=\lim _{\varepsilon \rightarrow 0}\left(\partial_{t} u_{\varepsilon}+\left(u_{\varepsilon} \bullet \nabla\right) u_{\varepsilon}+\left(v_{\varepsilon} \bullet \nabla\right) u_{\varepsilon}-\Delta u_{\varepsilon}+\nabla p_{\varepsilon}\right)=0 \text {. }
$$

It is clear that $\operatorname{div} b u_{\varepsilon}=0$ for $\varepsilon>0$ implies

$$
\begin{gathered}
\operatorname{div} b u=\operatorname{div} \lim _{\varepsilon \rightarrow 0} b u_{\varepsilon}=\lim _{\varepsilon \rightarrow 0} \operatorname{div} b u_{\varepsilon}=0, \\
\left.u\right|_{t=t_{0}}=\left.\lim _{\varepsilon \rightarrow 0} u_{\varepsilon}\right|_{t=t_{0}}=\lim _{\varepsilon \rightarrow 0} u_{\varepsilon 0}=u_{0}, \\
u(t, x)=\lim _{\varepsilon \rightarrow 0} u_{\varepsilon}(t, x)=0 \text { when } x \in \partial \Omega .
\end{gathered}
$$

Therefore $u, p$ satisfies (24).

Now we consider a special case (stationary Stokes equation). In [1] M.Braine and P.Gerard studied a scalar and a two-dimensional stationary Stokes equations perturbed by a drift in the sense of distributions. Under higher integrability conditions the Tartar approach based on the oscillations test functions method applies and leads to a limit equation with an extra zero-order term. However, the lack of integrability makes difficult the direct use of the Tartar method for the three-dimensional Stokes equations. But we can use the semigroup method to study the three-dimensional stationary Stokes equation in the usual sense $b D L_{2}(\Omega)$.

When a ship is sailing in the lake, the set dynamic speed is $v$. Due to the operation error, the actual dynamic speed is $v_{\varepsilon}(t, x), v_{\varepsilon}$ infinitely close to $v$, that is $\lim _{\varepsilon \rightarrow 0}\left\|v_{\varepsilon}-v\right\|_{b D L_{2}(\Omega)}=0$. How to calculate the relative speed of the ship, the speed of the combination of dynamic speed and lake speed? In order to keep the ship's speed stable, it is assumed that the ship's dynamic force $f_{\varepsilon}(t, x)$ makes the time-varying acceleration $\frac{\partial u_{\varepsilon}}{\partial t}$ and the spatial position dependent migration acceleration $\left(u_{\varepsilon} \bullet \nabla\right) u_{\varepsilon}$ to be zero. The transfer acceleration with the change of space 
position produced by ship dynamic force $f_{\varepsilon}(t, x)$ is $\left(v_{\varepsilon} \bullet \nabla\right) u_{\varepsilon}$. This acceleration $\left(v_{\varepsilon} \bullet \nabla\right)$ $u_{\varepsilon}$ counteracts the accelerations produced by the viscous force $\Delta u_{\varepsilon}$ and the pressure gradient force $\nabla p_{\varepsilon}$ such that $\frac{\partial u_{\varepsilon}}{\partial t}=0$ and $\left(u_{\varepsilon} \bullet \nabla\right) u_{\varepsilon}=0$. This is the result of zero resultant force of $f_{\varepsilon}, \Delta u_{\varepsilon}$ and $\nabla p_{\varepsilon}$. The relative speed of the ship is the homogenization of $u_{\varepsilon}, u=\lim _{\varepsilon \rightarrow 0} u_{\varepsilon}$. Our mathematical problem is as following

$$
\left\{\begin{array}{c}
\left(v_{\varepsilon} \bullet \nabla\right) u_{\varepsilon}-\Delta u_{\varepsilon}+\nabla p_{\varepsilon}=f_{\varepsilon}, x \in \Omega, t \in\left(t_{0}, T\right] \\
\operatorname{div}\left(b u_{\varepsilon}\right)=0, x \in \Omega \\
u_{\varepsilon}(t, x)=0, x \in \partial \Omega
\end{array}\right.
$$

From Theorem 2, Theorem 3 and Theorem 4 we have the following corollaries

Corollary 3. Suppose that for each $\varepsilon>0,\left(t_{0}, u_{\varepsilon 0}\right) \in U, b(x) \in C^{\infty}(\Omega), \quad v_{\varepsilon}, f_{\varepsilon}$ are Hölder continuous in $D L_{2}(\Omega)_{\frac{1}{2}}$ and in $D L_{2}(\Omega)$ respectively with exponent $\beta$, and $\left\|v_{\varepsilon}(t)\right\|_{D L_{2}(\Omega)_{\frac{1}{2}}} \leq L^{0}\left(t \in\left[t_{0}, T\right]\right)$ for some constant $L^{0}$. Then the viscous lake equations (25) has a unique smooth local strong solutions .

Corollary 4. Suppose that $\lim _{\varepsilon \rightarrow 0} v_{\varepsilon}=v$ in $b D L_{2}(\Omega)$. Then there exists $u \in b D L_{2}(\Omega)_{\frac{1}{2}}$ such that $\lim _{\varepsilon \rightarrow 0} u_{\varepsilon}=u$ in $b D L_{2}(\Omega)$.

Corollary 5. Assume that $\lim _{\varepsilon \rightarrow 0} v_{\varepsilon}=v, \lim _{\varepsilon \rightarrow 0} f_{\varepsilon}=f$ and there exists $p \in b D L_{2}(\Omega)$ such that $\lim _{\varepsilon \rightarrow 0} \nabla p_{\varepsilon}=\nabla p$, then $u, p$ satisfies

$$
\left\{\begin{array}{c}
-\Delta u+(v \bullet \nabla) u+\nabla p=f, x \in \Omega \\
\operatorname{div}(b u)=0, x \in \Omega \\
u=0, x \in \partial \Omega
\end{array} .\right.
$$

\section{References}

[1] Andras Batkai, Marjeta Kramar Fijavz, Abdelaziz Rhandi, Positive Operator Semigroups, Birkhauser (2017)

[2] Marc Braine,Patrick Gerard, A drift homogenization problem revisited, Ann.Sc.Norm.Super. 
Pisa.Cl.Sci (5)Vol.XI(2012),1-39

[3] D.Cioranescu, D.Donato, An Introduction to Homogenization, Oxford University Press, 1999

[4] H.Fujita, T.Kato, On the Navier-Stokes initial value problem I,Arch. Ration. Mech. Anal. 16(1964),269-315

[5] T.Kato, Strong $L_{p}$-solution of the Navier-Stokes equation in $R^{m}$, with application to weak solutions, Math. Z. 187(1984)471-480

[6] Engel Klaus-Jochen , Rainer Nagel. One-Parameter Semigroups for Linear Evolution Equations, Springer, (2000)

[7] E.Kreyszig, Introduction functional analysis with applications, John Wiley \&Sons (1978)

[8]Y. Giga, Analyticity of the semigroup generated by the Stokes operator on $L_{r}$-spaces, Math. Z. $178,297-329(1981)$

[8] Y.Giga,T.Miyakava, Solutions in $L_{r}$ of the Vavier-Stokesinitial value problem, Arch.Ration.Mech.Anal. 89(1985)267-281

[10] M.Otelbaev, Existence of a strong solution of the Navier-Stokes equation, Mathematical Journal, 13(4)(2013), 5-104

[11] A.Pazy, Semigroups of linear operators and applications to partial differential equations, Springer Verlag (1983, reprint in China in 2006)

[12] James C. Robinson, Infinite-Dimentional Dynamical Systems, Cambridge University Press, (2001)

[13] Walter Rudin, Principals of Mathematical Analysis, China Machine Press, 2004

[14] Walter Rudin, Real and Complex analysis, China Machine Press, 2004

[15] Veli B.Shakhmurov, Nonlocal Navier-Stokes problems in abstract function, Nonlinear Analysis:Real World Applications , 26(2015)19-43

[16] P.E. Sobolevskii, Study of the Navier-Stokes equations by the methods of the theory of parabolic equations in Banach spaces. Sov. Math. Dokl. 5, 720 - 723 (1964)

[17] Bilal Al Taki, Viscosity effect on the degenerate lake equations, Nonlinear Analysis, 148 (2017) 30-60

[18] Maoting Tong, Daorong Ton, A local strong solution of the Navier-Stokes problem in $L_{2}(\Omega)$, Journal of Mathematical Sciences: Advances and applications,

Volume No:62, Issue no:1,May(2020).1-19. Available at http://scientificadvances.co.in DOI: http://dx.doi.org/10.18642/jmsaa_7100122125 